\title{
Optimally Adaptive Power-Saving Protocols for Ad Hoc Networks Using the Hyper Quorum System
}

\author{
Shan-Hung Wu, Ming-Syan Chen, Fellow, IEEE, ACM, and Chung-Min Chen
}

\begin{abstract}
Quorum-based power-saving (QPS) protocols have been proposed for ad hoc networks (e.g., IEEE 802.11 ad hoc mode) to increase energy efficiency and prolong the operational time of mobile stations. These protocols assign to each station a cycle pattern that specifies when the station should wake up (to transmit/receive data) and sleep (to save battery power). In all existing QPS protocols, the cycle length is either identical for all stations or is restricted to certain numbers (e.g., squares or primes). These restrictions on cycle length severely limit the practical use of QPS protocols as each individual station may want to select a cycle length that is best suited for its own need (in terms of remaining battery power, tolerable packet delay, and drop ratio). In this paper, we propose the notion of hyper quorum system (HQS) - a generalization of QPS that allows for arbitrary cycle lengths. We describe algorithms to generate two different classes of HQS given any set of arbitrary cycle lengths as input. We also describe how to find the optimal cycle length for a station to maximize energy efficiency, subject to certain performance constraints. We then present analytical and simulation results that show the benefits of HQS-based power-saving protocols over the existing QPS protocols. The HQS protocols yield up to $41 \%$ improvement in energy efficiency under heavy traffic loads while eliminating more than $\mathbf{9 0 \%}$ delay drops under light traffic loads.
\end{abstract}

Index Terms-Power saving, quorum systems, wireless ad hoc networks.

\section{INTRODUCTION}

A D HOC network technologies and standards, such as IEEE 802.11 's ad hoc mode, allow the quick setup of a wireless network among a group of mobile stations, where the stations communicate with each other either directly or indirectly through multiple hops, without the aid of an infrastructure (e.g., cables, access points or base stations). The mobile stations (devices) in the network usually rely on batteries as the power source; thus, ensuring energy efficiency during ad hoc communication is essential to prolonging the operational time of the devices.

Energy conservation could be achieved at different layers with different techniques. When a station is not transmitting, the transceiver at PHY layer persists in idle mode and continuously listens for incoming transmissions. Studies [11], [15] find that the energy consumed by a wireless module in listening to

Manuscript received March 06, 2011; revised February 14, 2012; accepted May 18, 2012; approved by IEEE/ACM TRANSACTIONS ON NETWORKING Editor S. Ramasubramanian. Date of publication January 20, 2014; date of current version February 12, 2014.

$\mathrm{S} .-\mathrm{H}$. Wu is with the Department of Computer Science, National Tsing Hua University, Hsinchu, Taiwan (e-mail: shwu@cs.nthu.edu.tw).

M.-S. Chen is with the Department of Electrical Engineering, National Taiwan University, Taipei, Taiwan (e-mail: mschen@cc.ee.ntu.edu.tw).

C.-M. Chen is with Telcordia Technologies Applied Research, Piscataway, NJ 08854 USA (e-mail: chungmin@research.telcordia.com).

Digital Object Identifier 10.1109/TNET.2013.2296614 the network is only slightly lower than that of transmitting and receiving data. Therefore, if there are seldom transmissions destined for the station, idle listening would waste a significant amount of energy. To address this problem, a power management policy is usually adopted at MAC layer that, instead of idle listening, allows a station to sleep (or doze) - to suspend the transceiver-when there is no data transmission.

The IEEE 802.11 Power-Saving (PS) mode [10] includes a mechanism that allows a station to go into sleep when there is no data transmission. The standard divides the timeline evenly into beacon intervals and requires all stations to wake up at the beginning of each beacon interval to check if there are data transmission requests from other stations (for this purpose, all stations must have their clocks synchronized). A station will goes into sleep for the rest of a beacon interval if there is no data transmission request from other stations destined for the station. Nonetheless, studies [13], [27] have shown that the IEEE 802.11 PS mode is not energy-efficient under light data traffic loads because a station still needs to remain awake for a short time window at the beginning of each beacon interval. To address this problem, recent work [12], [22], [26] has explored the quorum-based power-saving (QPS) protocols that require a station to wake up only for certain beacon intervals (instead of all beacon intervals as in 802.11 PS mode). Given an integer $n$, a quorum system defines, for each station in the network, a cycle pattern (or cycle for short) that specifies the awake/sleep schedule for $n$ continuous beacon intervals. This pattern repeats every $n$ beacon intervals, and therefore $n$ is called the cycle length. The QPS protocols require a station to remain awake for only $O(\sqrt{n})$ beacon intervals per cycle and guarantees that any two stations will overlap in at least one awakened beacon interval within each cycle. A QPS system also ensures that the collective awakened beacon intervals from all stations are distributed uniformly within a cycle so that the bandwidth of the network can be fully utilized. It has been shown that QPS protocols render much better power saving than IEEE 802.11 PS mode under light traffic loads.

In QPS protocols, there is a tradeoff when it comes to selecting a value for the cycle length $n$-the larger the value of $n$, the more the power saving, yet the longer the buffering delay at the sender as data must be buffered until the recipient wakes up. Requiring all stations to use the same value of cycle length is problematic as individual stations may have their own delay requirement on data communications and constraint of remaining battery power. This consideration leads to a number of recent works [5], [7] that allow the stations to use different values of cycle length $n$, with certain restrictions. In AQEC [5], $n$ is required to be a square; while in AAPM [7], $n$ must be a 
prime number. We call these QPS systems semi-adaptive in the sense that they allow stations to use different cycle lengths but only with certain values. In reality, both these systems render a very limited selection for the values of $n$, as the cycle length must be small (typically, $n \leq 25$ ) due to the consideration of node mobility and/or route advertisement interval at the network layer [21]. For example, suppose a routing advertisement packet at Network layer needs to be sent within every $2 \mathrm{~s}$, and the duration of a beacon interval is $100 \mathrm{~ms}$. The neighbor discovery delay cannot be longer than 20 beacon intervals. The $O(n)$ delay bound [5] of AQEC implies that there are only three cycle lengths $(4,9,16)$ selectable for a station. This number is far less than the optimal case where all 19 cycle lengths (from 2 to 20) are available. Since the station needs to remain awake in at least $O(\sqrt{n})$ beacon intervals every cycle, it can easily miss the chance of picking the optimal cycle length that consumes the least energy while giving satisfactory delay. Studies [2], [14], [28] have shown that devices can end up consuming excessive energy if any inappropriate cycle length is used. This urges the need for a new power management solution that offers more flexibility in the selection of cycle lengths.

\section{A. Highlight of Our Contributions}

In this paper, we propose and define the notion of hyper quorum system (HQS) that generalizes traditional quorum-based systems to allow for arbitrary cycle lengths for different mobile stations while guaranteeing overlap of awakened periods between any two stations. We note that HQS is "fully adaptive" in the sense that a station can select any value of cycle length that is best suited to its own requirements in terms of packet delay and power constraint. Our major contributions include the following.

- We propose two constructive algorithms to build HQS schemes, one based on the semi-adaptive grid QPS [22] and one based on the difference set [17], in $O(1)$ time. To the best of our knowledge, these are the first known fully adaptive quorum-based power management protocols for ad hoc networks. Furthermore, these two schemes are interoperable and can respectively be adopted by mobile stations that are either delay-sensitive or require best-effort traffic.

- We derive an analytical model that characterizes the performance of a QPS protocol in terms of energy efficiency, packet delay, and drop ratio. This model is validated by extensive simulations and can be used by individual stations to determine the optimal cycle length to use, with the objective to maximize energy efficiency subject to given constraints in delay and drop ratio.

- We show that the HQS-based power management protocols can be applied to either the synchronous or asynchronous environments where the beacon intervals on stations are aligned or not, respectively. This eliminates the need of clock synchronization, which could be cost-prohibitive when a large number of stations is involved.

- Experimental results show that the HQS-based power management protocol may offer significantly better performance than traditional QPS protocols. In particular, it yields up to $41 \%$ improvement in energy efficiency under heavy traffic loads while eliminating more than $90 \%$ packet delay drops under light traffic loads.

The rest of this paper is organized as follows. In Section II, we review existing power management protocols for ad hoc networks. Section III formally defines the notion of HQS and describes two HQS constructing schemes. The power management protocol based on HQS is also elaborated. In Section IV, we present an analytical model of the performance of HQS and derive the optimal cycle length for each station subject to certain conditions. Section V presents the performance evaluation of our protocols based on both analytical and simulation results. Section VI concludes the paper.

\section{PRELIMINARIES}

In this section, we review existing power management protocols for ad hoc networks.

\section{A. IEEE Power-Saving Mode}

IEEE 802.11 [10] is currently one of the most popular MAC standards for wireless ad hoc networks. The operation of IEEE 802.11 PS mode is shown in Fig. 1(a). The time axis is divided evenly into beacon intervals, and all stations are synchronized to align on these intervals. At the beginning of each beacon interval is an Announcement Traffic Indication Message (ATIM) window. Each station is required to wake up and remain awake during the entire ATIM window.

If a station, say $H_{1}$, intends to transmit data to a destination, say $H_{0}$ [Fig. 1(a) (1)], it first unicasts an ATIM frame to $H_{0}$ during the ATIM window [Fig. 1(a) (2)]. $H_{0}$, upon receiving the ATIM frame, will send back an acknowledgment. After this ATIM notification procedure, both $H_{0}$ and $H_{1}$ will keep awake for the rest of the beacon interval, during which the Distributed Coordination Function (DCF) mechanism (i.e., RTS, CTS, and random backoff) [10] will be initiated to transmit the data [Fig. 1(a) (3)] while avoiding collisions.

If a station neither sends out nor receives an ATIM notification during the ATIM window, it may enter the doze mode (that is, to sleep) for the rest of the beacon interval.

In IEEE 802.11 PS mode, the Target Beacon Transmission Time (TBTT) on all stations should be aligned to ensure the overlap of ATIM windows. To synchronize the timer, all stations contend to send a beacon frame, which carries timer information, at the beginning of a beacon interval. Upon hearing the first beacon, each station synchronizes its clock with the carried information and cancels its own beacon transmission.

\section{B. Quorum-Based Power-saving Protocols}

Existing QPS protocols for ad hoc networks can be classified into two categories: the static and semi-adaptive protocols, as shown in Table I. The static QPS protocols require all stations to use the same cycle length in order to communicate with each other. Recently, the semi-adaptive QPS protocols [5]-[7] were proposed that allow stations to choose different cycle

\footnotetext{
${ }^{1}$ Note that in the situation where data transmission cannot complete within a single beacon interval (due to collisions or large data volume), $H_{1}$ can set the more-data bit (in frame header) true telling $H_{0}$ to remain awake through the successive beacon interval, and then the data transmission continues [10].
} 


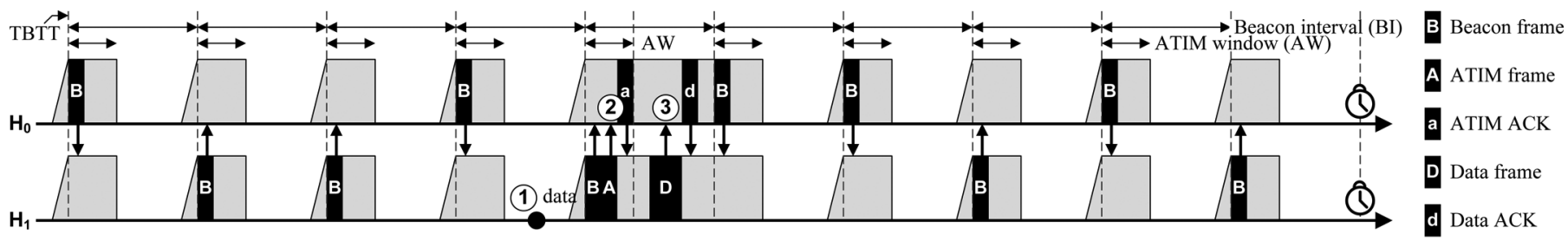

(a)

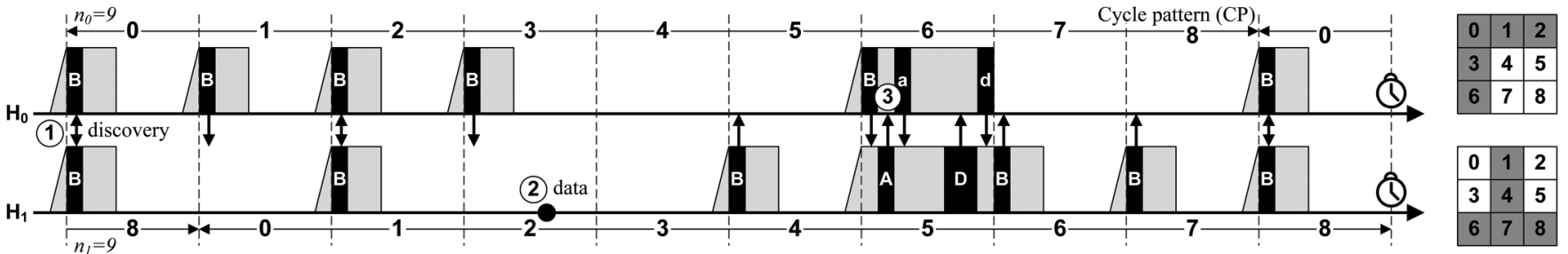

(b)

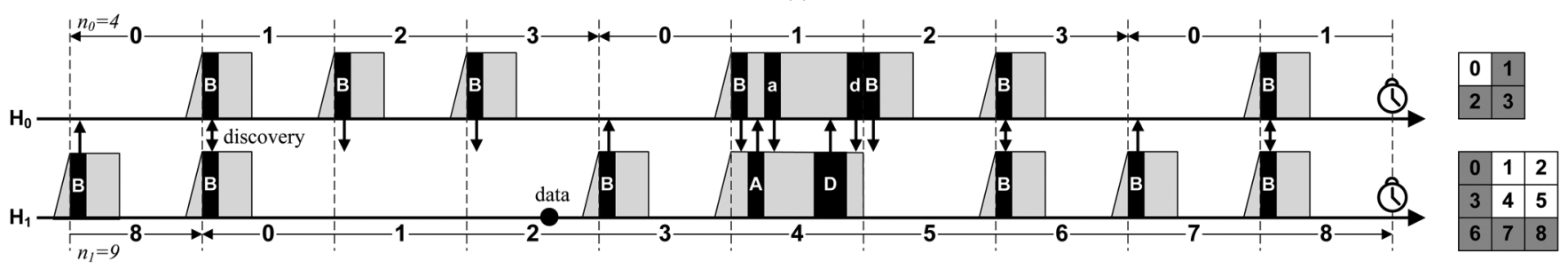

(c)

Fig. 1. Existing ad hoc power management protocols. (a) IEEE PS mode. (b) Static grid/torus-based quorum systems. (c) AQEC with semi-adaptivity.

TABLE I

CATEgorization of the Quorum-BAsed Power Management Protocols

\begin{tabular}{c|ccc} 
Environments\Adaptivity & Static & Semi-Adaptive & Adaptive \\
\hline Synchronous & {$[23],[25]$} & {$[5]$} & None \\
Asynchronous & {$[12],[27]$} & {$[6],[7]$} & None
\end{tabular}

lengths from a set of numbers satisfying certain restrictions (e.g., primes).

Existing QPS protocols target on either the synchronous or asynchronous environments, where the timers of stations are assumed to be synchronized or not, respectively. Synchronous QPS protocols [5], [22], [24] rely on a timer synchronization mechanism to ensure the overlap of awakened periods between each pair of stations. The asynchronous QPS protocols [6], [12], [26] ensure the overlap of awakened periods even when there is no timer synchronization mechanism. The latter protocols are more scalable to large numbers of stations as timer synchronization is costly in such situations. This advantage, however, comes at the expense of less energy efficiency as a station will need to stay awake for the entirety of a beacon interval for which the station is scheduled to wake up (in contrast, in synchronous QPS, a station only needs to stay awake during the ATIM windows of those beacon intervals).

In the following, we briefly summarize the static, grid-based QPS protocols [12], [22] and the semi-adaptive AQEC protocol [5] as they are relevant to our study.

Grid-Based QPS Protocols: Fig. 1(b) illustrates the awake/ sleep schedules of two stations, $H_{0}$ and $H_{1}$, which are defined by a grid quorum scheme with cycle length $n=9$. The grid quorum scheme organizes every $n$ continuous beacon intervals into an $\sqrt{n} \times \sqrt{n}$ array and numbers them from 0 to $n-1$ in a row-major manner. It defines a quorum as a set containing the numbers along an arbitrary row and an arbitrary column in the array [e.g., $\{0,1,2,3,6\}$ or $\{1,4,6,7,8\}$ as shaded in Fig. 1(b)]. Each station, by using this scheme, is able to obtain its own quorum of quorum size (i.e., cardinality) $2 \sqrt{n}-1$. For all beacon intervals whose numbers are specified in the quorum, the station will remain awake during the ATIM windows (as in IEEE 802.11 PS mode). For beacon intervals whose numbers do not appear in the quorum, the station will sleep without even awaking for the ATIM windows. Such a schedule repeats every $n$ beacon intervals and is called the cycle pattern (or cycle for short). We denote the duration of the ATIM window and beacon interval as $\bar{A}$ and $\bar{B}$, respectively. This scheme gives the lowest duty cycle (i.e., the portion of time a station remains awake) $\frac{(2 \sqrt{n}-1) \bar{A}}{n \bar{B}}$. Apparently, the larger the cycle length $n$, the more the power saving.

In QPS protocols, a beacon frame should carry additional information about the schedule, including the adopted quorum and the number of current beacon interval, etc. Unlike the IEEE 802.11 PS mode where a station should cancel its own beacon transmission upon hearing the first beacon frame, each station should persist its beacon transmission (even when the others' beacons are heard) to claim its own schedule.

As we can see in Fig. 1(b), each quorum in the grid quorum scheme intersects with any other quorum in two elements. This implies that the ATIM windows between stations overlap twice per cycle. Once beacon frames are exchanged at an overlapped period [Fig. 1(b) (1)], stations $H_{0}$ and $H_{1}$ are able to discover each other - that is, to receive and keep one another's schedule and to predict the next coming of ATIM window at the other party. Suppose $H_{1}$ has data for $H_{0}$ [Fig. 1(b) (2)]; it buffers 
the data and waits for the next ATIM window of $H_{0}$. When $H_{0}$ wakes up [Fig. 1(b) (3)], $H_{1}$ unicasts an ATIM frame to $H_{0}$ and starts the notification and data transmission procedures as described previously in the IEEE 802.11 PS mode.

It is important to note that the grid quorum scheme ensures the overlaps of ATIM windows even when the numbering of beacon interval shifts between stations. For example, as we can see in Fig. 1(b) $H_{0}$ 's clock leads $H_{1}$ 's clock by one beacon interval. The quorum adopted by $H_{0}$, from $H_{1}$ 's point of view, becomes $\{0-1 \bmod 9,1-1 \bmod 9,2-1 \bmod 9,3-1 \bmod 9,6-$ $1 \bmod 9\}=\{8,0,1,2,5\}$. We can easily verify that the rotated schedule of $H_{0}$ still overlaps twice per cycle with that of $H_{1}$. This nice property is due to the fact that the intersections between $H_{0}$ 's and $H_{1}$ 's quorums are shift-invariant. ${ }^{2}$ Apparently, only those quorum schemes producing shift-invariant quorums can be used in a QPS protocol. The lower bound of the size of these shift-invariant quorums is $\sqrt{n}$ [12].

The power-saving advantage provided by QPS protocols comes at the price of delay. Such delay includes the data buffering time, i.e., the duration between a packet arrival (on a sending station) and its start of DCF. As we can see in previous examples, the data buffering time in grid-based QPS protocol is at most $O(\sqrt{n}) \bar{B}$. Besides, two adjacent stations may not be able to discover each other until a cycle passes by. The neighbor discovery time, i.e., the time for a station to discover its new neighbor, is therefore $O(n) \bar{B}$ in the worst case.

Semi-Adaptive Protocols: The static, grid-based quorum scheme is extended to a semi-adaptive one in AQEC [5], where each station is free to choose a cycle length, given that it is a square number. Fig. 1(c) illustrates an example where $H_{0}$ and $H_{1}$ adopt cycle patterns with different square lengths $n=4$ and $n^{\prime}=9$, respectively. It can be shown that $H_{0}$ and $H_{1}$ are guaranteed to receive one another's beacon frame at least once every $\sqrt{n_{\min }}+n_{\max }-1$ beacon intervals, where $n_{\min }=\min \left\{n, n^{\prime}\right\}$ and $n_{\max }=\max \left\{n, n^{\prime}\right\}$. This implies that the neighbor discovery time is $O\left(\sqrt{n_{\min }}+n_{\max }-1\right) \bar{B}$. Other schemes, which are based on the Finite Projective Plane (FPP) [7] or Symmetric Block Design [6], give similar flexibility in choosing $n$ except that $n$ must be a prime [7] or compound of primes [6]. Note that the FPP-based scheme has one serious drawback as compared to the grid scheme-it does not give bounded neighbor discovery time. Besides, scheme [7] works only when $n \leq 47$.

As mentioned earlier, requiring the cycle lengths to be squares or primes could be too much a stringent requirement, ${ }^{3}$ as in practice the cycle lengths are usually smaller than 25 due to the fact that stations need to discover/update its neighbors once within a certain period (e.g., a route advertisement interval), which is usually no longer than 2 or $3 \mathrm{~s}$. This makes semi-adaptive protocols hard to be applied to the real networks where the node mobility and/or routing requirements are present. ${ }^{4}$ Studies [2], [14], and [28] have shown that a PS

\footnotetext{
${ }^{2} \mathrm{~A}$ quorum scheme that guarantees the shift-invariant intersection is called cyclic. We will formally define the cyclic property in Section III.

${ }^{3}$ In the simulation part of study [5], AQEC provides only four practical choices (i.e., 4, 9, 16, and 25) for cycle lengths.

${ }^{4}$ Although it is understandable that the study [6] only considered the delaytolerant networks.
}

station compromising on a suboptimal cycle length may end up consuming more energy than a non-PS station because of the excessive packet drops and retransmissions. Apparently, to allow a fine-tunable cycle length on each station is a key to success for a QPS protocol.

\section{HYPER QUORUM SYSTEM}

This section defines the notion of hyper quorum system and describes algorithms to construct two different classes of HQS. HQS is a generalization of traditional quorum systems [3] where stations may obtain cycle patterns of arbitrary lengths that best suit their requirements.

The advantage offered by HQS is twofold. First, HQS allows more energy saving on those stations having critical battery power or light traffic load. This overall prolongs the lifetime of a network. In addition, HQS gives controllable delay that is able to meet application requirements. This avoids serious performance degradation and energy waste on packet retransmissions as induced by traditional power management protocols. Note that this advantage becomes significant when all peers in a network have distinct requirements of energy consumption and delay, which is usually true due to the number of hops, battery life, mobility, and running applications.

\section{A. Definitions and Fundamentals}

Consider the sets in which each element denotes a number of beacon interval. The following definitions are based on [3] and [17].

Definition 1 ( $n$-Coterie): Given an integer $n$ and a universal set $U=\{0,1, \ldots, n-1\}$ over the modulo- $n$ plane. Let $X$ be a set of nonempty subsets of $U$. We call $X$ an n-coterie if and only if for all $Q, Q^{\prime} \in X, Q \cap Q^{\prime} \neq \varnothing$.

For example, consider $X=\{\{0,1,2,3,6\},\{1,4,6,7,8\}\}$ in Fig. 1(b). For any pair of elements $Q=\{0,1,2,3,6\}$ and $Q^{\prime}=\{1,4,6,7,8\}, Q \cap Q^{\prime} \neq \varnothing$. Therefore, $X$ is a 9-coterie. Conventionally, a coterie $X$ is termed a quorum system, and the elements of $X$ (i.e., $Q$ ) are called the quorums.

Not every quorum system is applicable to a QPS protocol [12]. In a QPS protocol, two quorums must intersect even when one "shifts." This leads to the following definitions.

Definition $2((n, l)$-Cyclic Set): Given integers $n$ and $l$, where $0 \leq l \leq n-1$. Let $Q$ be a subset of $U, U=\{0,1, \ldots, n-$ $1\}$. We call $C_{n, l}(Q)$ an $(n, l)$-cyclic set of $Q$ if and only if $C_{n, l}(Q)=\{(q+l) \bmod n: \forall q \in Q\}$.

For convenience, we denote a group of cyclic set as $C_{n}(Q)=$ $\left\{C_{n, l}(Q): \forall l\right\}$.

Definition 3 (n-Cyclic Quorum System): Given an integer $n$ and a universal set $U=\{0,1, \ldots, n-1\}$ over the modulo- $n$ plane. Let $X=\left\{Q_{0}, Q_{1}, \ldots, Q_{d-1}\right\}, d \in \mathbb{Z}$, be a set of nonempty subsets of $U$. We call $X$ an $n$-cyclic quorum system if and only if the set $C_{n}\left(Q_{0}\right) \cup C_{n}\left(Q_{1}\right) \cup \cdots \cup C_{n}\left(Q_{d-1}\right)$ is an $n$-coterie.

For example, the set $\{\{0,1,2,3,6\},\{1,4,6,7,8\}\}$ also forms a 9-cyclic quorum system, as every element pair in

$$
\begin{aligned}
\{\{0,1,2,3,6\},\{1,2,3,4,7\}, \ldots,\{8,0,1,2,5\}\} \\
\cup\{\{1,4,6,7,8\},\{2,5,7,8,0\}, \ldots,\{0,3,5,6,7\}\}
\end{aligned}
$$


intersects. The cyclic property ensures the shift-invariant intersection between $\{0,1,2,3,6\}$ and $\{1,4,6,7,8\}$. Recall in Fig. 1 (b) $H_{0}$ 's clock leads $H_{1}$ 's clock by one beacon interval. From $H_{1}$ 's point of view, the quorum adopted by $H_{0}$ equals $\{8,0,1,2,5\}$, which, by definition, belongs to $C_{9}(\{0,1,2,3,6\})$ and still intersects with $H_{1}$ 's quorum $\{1,4,6,7,8\}$. The above definition serves as the basis for most quorum schemes used in existing QPS protocols [5], [7], [12], [22], [26].

In the following, we generalize the definition of cyclic coterie to the notion of hyper quorum system. First, we define a useful notation.

Definition $4((n, m, l)$-Revolving Set): Given integers $n, m$, and $l$, where $0 \leq l \leq n-1$. Let $Q$ be a subset of $U, U=$ $\{0,1, \ldots, n-1\}$. We call $R_{n, m, l}(Q)$ an $(n, m, l)$-revolving set of $Q$ if and only if $R_{n, m, l}(Q)=\{(q+i n)-l: 0 \leq(q+i n)-l \leq$ $m-1, \forall q \in Q, i \in \mathbb{Z}\}$.

Intuitively, $R_{n, m, l}(Q)$ is a projection of $Q$ from the modulo- $n$ plane onto the modulo- $m$ plane with an index shift $l$. For example, consider two quorums $Q_{0}=\{1,2,3\}$ and $Q_{1}=\{0,3,6,7,8\}$ shown in Fig. 1(c), which are subsets of $U_{0}=\{0,1,2,3\}$ and $U_{1}=\{0,1, \ldots, 8\}$, respectively. Given two shift indices $l_{0}=2$ and $l_{1}=1$, we may project these two sets from the modulo-4 and -9 planes onto the same modulo-10 plane by using $R_{4,10,2}\left(Q_{0}\right)=\{0,1,3,4,5,7,8,9\}$ and $R_{9,10,1}\left(Q_{1}\right)=\{2,5,6,7,8\}$, respectively. Note that both $R_{4,10,2}\left(Q_{0}\right)$ and $R_{9,10,1}\left(Q_{1}\right)$ are subsets of a new universal set $U^{\prime}=\{0,1, \ldots, 9\}$. For convenience, we denote a group of revolving sets as $R_{n, m}(Q)=\left\{R_{n, m, l}(Q): \forall l\right\}$.

Definition $5\left(\left(n_{0}, n_{1}, \ldots, n_{d-1} ; m\right)\right.$-Hyper Quorum System): Given integers $n_{0}, n_{1}, \ldots, n_{d-1}$ and $m$, where $d \in \mathbb{Z}$. Let $X=\left\{Q_{0}, Q_{1}, \ldots, Q_{d-1}\right\}$ be a set with the element $Q_{i}$, $0 \leq i \leq d-1$, a nonempty subset of the universal set $U_{i}=\left\{0,1, \ldots, n_{i}-1\right\}$ over the modulo- $n_{i}$ plane. We call $X$ an $\left(n_{0}, n_{1}, \ldots, n_{d-1} ; m\right)$-hyper quorum system if and only if the set $R_{n_{0}, m}\left(Q_{0}\right) \cup R_{n_{1}, m}\left(Q_{1}\right) \cup \cdots \cup R_{n_{d-1}, m}\left(Q_{d-1}\right)$ forms an $m$-coterie.

Basically, a hyper quorum system guarantees the intersection between the projections of quorums over a plane, as depicted in Fig. 2. Following the example shown in Fig. 1(c) where $Q_{0}=\{1,2,3\}$ and $Q_{1}=\{0,3,6,7,8\}$. Given a reference point of time $t$. Suppose at time $t$, the stations $H_{0}$ and $H_{1}$ are in their second and first beacon interval, respectively. Then, $H_{0}$ and $H_{1}$ are guaranteed to overlap in at least one awake beacon interval within the 10 beacon intervals after $t$ since $R_{4,10,2}\left(Q_{0}\right) \cap$ $R_{9,10,1}\left(Q_{1}\right) \neq \varnothing$. Actually, we may easily verify that given any reference point of time where $H_{0}$ and $H_{1}$ are in their $l_{0}$ th and $l_{1}$ th beacon intervals, respectively, $H_{0}$ and $H_{1}$ are guaranteed to overlap within 10 beacon intervals. Formally, we have $R_{4,10, l_{0}}\left(Q_{0}\right) \cap R_{9,10, l_{1}}\left(Q_{1}\right) \neq \varnothing$ for all $l_{0}$ and $l_{1}, 0 \leq l_{0} \leq 3$ and $0 \leq l_{1} \leq 8$. Therefore, the set $\{\{1,2,3\},\{0,3,6,7,8\}\}$ is a $(4,9 ; 10)$-hyper quorum system. In Definition $5, X$ is said to be $d$-dimensional, and the elements of $X$ are termed quorums.

Notice that a revolving set $R_{n, m, l}(Q)$ degenerates into a cyclic set $C_{n,(-l \bmod n)}(Q)$ when $m=n$. An $\left(n_{0}, n_{1}, \ldots, n_{d-1} ; m\right)$-hyper quorum system degenerates into a traditional $n$-cyclic quorum system when $n=n_{0}=n_{1}=\cdots=n_{d-1}=m$.

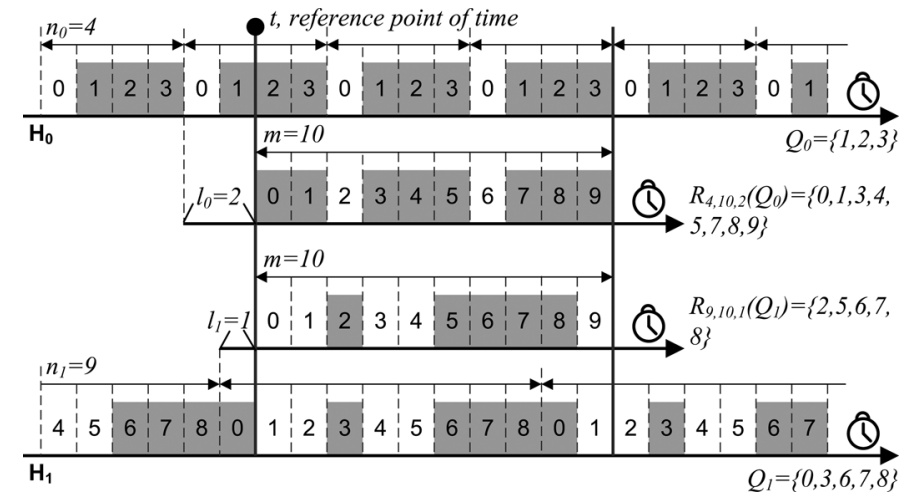

Fig. 2. Rather than ensuring the intersection between quorums, the hyper quorum system guarantees the intersection between "projections" of quorums over a modulo- $m$ plane.

By following the example given in Fig. 2, we may observe that, first, HQS ensures a shift-invariant intersection, therefore supports any QPS protocol naturally. Suppose $H_{0}$ 's clock is shifted 1 beacon interval ahead. The projected quorum of $H_{0}$ at time $t$ becomes $R_{4,10,(2+1)}\left(Q_{0}\right)$, which, by definition, belongs to $R_{4,10}\left(Q_{0}\right)$ and still intersects with $R_{9,10,1}\left(Q_{1}\right)$. Second, starting from any reference point of time, $H_{0}$ and $H_{1}$ are able to exchange their beacon frames within $m$ beacon intervals. An $\left(n_{0}, n_{1}, \ldots, n_{d-1} ; m\right)$-hyper quorum system guarantees the worst-case neighbor discovery time $O(m) \bar{B}$. Note that the FPP-based scheme [7] also gives an HQS. However, it does not guarantee a specific value of $m$ given $n_{0}$ to $n_{d-1}$. The neighbor discovery time is not predictable with this scheme.

It remains a challenging issue to efficiently construct an HQS given arbitrary modulo planes and arbitrary dimensions. Current schemes [5], [7] generate an HQS only when $n_{0}, n_{1}, \ldots, n_{d-1}$ are either squares or primes. Moreover, the HQS proposed in [7] is constructed by exhaustive searches. Next, we present algorithms that can systematically generate two different classes of HQS in $O(1)$ time over arbitrary modulo planes.

\section{B. Constructing Schemes for $H Q S$}

We present two HQS constructing schemes that offer flexibility in supporting arbitrary values of $n$ and $d$ while keeping the quorum size close to the lower bound $\sqrt{n}$ [12].

Extended Grid (EG) Scheme: Consider $d$ integers $n_{0}, n_{1}, \ldots, n_{d-1}$, where $n_{0}<n_{1}<\cdots<n_{d-1}$. Let $\phi_{i}=\min \left\{\left\lfloor\sqrt{n_{i}}\right\rfloor,\left[\sqrt{\left(n_{d-1}+1\right) / 2}\right\rceil\right\}$ and $q_{i}=\left\lfloor n_{i} / \phi_{i}\right\rfloor$ for all $i, 0 \leq i \leq d-1$. We define an extended grid quorum $G_{i}$ over the modulo- $n_{i}$ plane as follows:

$$
G_{i}=\left\{0,1, \ldots, \phi_{i}-1, g_{1}, g_{2}, \ldots, g_{q_{i}-1}\right\}
$$

where $\phi_{i}-1<g_{1} \leq 2 \phi_{i}-1$ and $0<g_{j+1}-g_{j} \leq \phi_{i}$ for all $j$, $1 \leq j \leq q_{i}-2$. Essentially, $G_{i}$ contains $\phi_{i}$ continuous elements from 0 to $\phi_{i}-1$, followed by $q_{i}-1$ interspaced elements with mutual distances less than or equal to $\phi_{i}$. Note that with the above definition, $G_{i}$ is not unique.

The name of this scheme comes from the fact that when $n_{i}$ is a square and $g_{j+1}-g_{j}=\phi_{i}, G_{i}$ degenerates into a grid-based quorum. For example, let $d=2, n_{0}=9$, and $n_{1}=20$. By 
fixing $g_{j+1}-g_{j}=3$, we have $G_{0}=\{0,1,2,5,8\}$, a quorum comprising a column and a row in the $3 \times 3$ grid.

It can be shown that $\left\{G_{0}, G_{1}, \ldots, G_{d-1}\right\}$ forms a $d$-dimensional HQS for some value of $m$. Instead of proving this, we will show that any pair of $G_{i}$ and $G_{j}$ forms a $\left(n_{i}, n_{j} ; m\right)$-hyper quorum system with a better bounded value of $m$. First, we define the heads of a revolving set $R_{n, m, l}(Q)$ to be those elements projected from the smallest element in $Q$ (note that there could be none, or more than one head). In the previous example shown in Fig. 2, the elements 3 and 7 are heads of $R_{4,10,2}\left(Q_{0}\right)$, and 8 is the head of $R_{9,10,1}\left(Q_{1}\right)$.

Theorem 6: Given $d$ integers $n_{0}, n_{1}, \ldots, n_{d-1}$, where $n_{0}<$ $n_{1}<\cdots<n_{d-1}$. For any $i$ and $j, 0 \leq i \leq j \leq d-1$, the set $\left\{G_{i}, G_{j}\right\}$ forms an $\left(n_{i}, n_{j} ; \phi_{i}+n_{j}-1\right)$-hyper quorum system.

Proof: Let $m=\phi_{i}+n_{j}-1$, we show that $R_{n_{i}, m, a}\left(G_{i}\right) \cap$ $R_{n_{j}, m, b}\left(G_{j}\right) \neq \varnothing$ for all $a$ and $b, 0 \leq a \leq n_{i}$ and $0 \leq b \leq$ $n_{j}$. Recall that the heads of $R_{n_{j}, m, b}\left(G_{j}\right)$ are the elements projected from the first element 0 in $G_{j}$. Let $h$ be the first head in $R_{n_{j}, m, b}\left(G_{j}\right)$. Since $m \geq n_{j}, h$ exists and $h \leq n_{j}-1$. If $h$ is included in $R_{n_{i}, m, a}\left(G_{i}\right)$, we finish the proof. Otherwise, consider two elements $s$ and $t$ in $R_{n_{i}, m, a}\left(G_{i}\right)$ such that $s<h<t$. By definition of $G_{i}$, any two interspaced elements in $R_{n_{i}, m, a}\left(G_{i}\right)$ must have mutual distance less than or equal to $\phi_{i}$. We have $t \leq s+\phi_{i}$, leading to $t \leq h+\phi_{i}-1 \leq\left(n_{j}-1\right)+\phi_{i}-1=m-1$. The element $t$ exists in $R_{n_{i}, m, a}\left(G_{i}\right)$. On the other hand, by definition of $G_{j}, h$ is a head of $R_{n_{j}, m, b}\left(G_{j}\right)$ implies that there exist $\phi_{j}-1$ continuous elements after $h$ in $R_{n_{j}, m, b}\left(G_{j}\right)$. Since $t \leq h+\phi_{i}-1 \leq h+\phi_{j}-1$, the element $t$ must also be included in $R_{n_{j}, m, b}\left(G_{j}\right)$. We have $R_{n_{i}, m, a}\left(G_{i}\right) \cap R_{n_{j}, m, b}\left(G_{j}\right) \neq \varnothing . \square$

Note that the above theorem applies to the grid-based scheme (e.g., AQEC [5]) also. Before this paper, quorum schemes for QPS protocols are proposed in an ad hoc manner. The HQS lays the fundamentals of these quorum schemes by identifying the combinatorial properties that need to be satisfied to make a scheme useful to a QPS protocol.

Difference Set (DS) Scheme: Consider $d$ integers $n_{0}, n_{1}, \ldots, n_{d-1}$, where $n_{0}<n_{1}<\cdots<n_{d-1}$. Let $\phi=\left\lceil\sqrt{\left(n_{d-1}+1\right) / 2}\right\rceil$ and $q_{i}=\left\lceil\frac{n_{i}+1}{2 \phi}\right\rceil$ for all $i$, $0 \leq i \leq d-1$. We define another quorum $D_{i}$ over the modulo- $n_{i}$ plane as follows:

$$
D_{i}=\left\{0,1, \ldots, \phi-1, d_{1}, d_{2}, \ldots, d_{q_{i}-1}\right\}
$$

where $\phi-1<d_{1} \leq 2 \phi-1,0<d_{j+1}-d_{j} \leq \phi$ for all $j, 1 \leq j \leq q_{i}-2$, and $d_{q_{i}-1} \geq\left(n_{i}-1\right) / 2$. Basically, $D_{i}$ contains $\phi$ continuous elements from 0 to $\phi-1$, followed by $q_{i}-1$ interspaced elements with mutual distances less than or equal to $\phi$. Note, again, that $D_{i}$ is not unique with the above definition, and $D_{i}$ is a difference set [17].

Theorem 7: Given $d$ integers $n_{0}, n_{1}, \ldots, n_{d-1}$, where $n_{0}<$ $n_{1}<\cdots<n_{d-1}$. Let $\phi=\left\lceil\sqrt{\left(n_{d-1}+1\right) / 2}\right\rceil$. For any $i$ and $j, 0 \leq i \leq j \leq d-1$, the set $\left\{D_{i}, D_{j}\right\}$ forms an $\left(n_{i}, n_{j} ;\left\lfloor\left(n_{i}-1\right) / 2\right\rfloor+n_{j}+\phi-1\right)$-hyper quorum system.

Proof: Let $m=\left\lfloor\left(n_{i}-1\right) / 2\right\rfloor+n_{j}+\phi-1$, we show that $R_{n_{i}, m, a}\left(D_{i}\right) \cap R_{n_{j}, m, b}\left(D_{j}\right) \neq \varnothing$ for all $a$ and $b, 0 \leq a \leq n_{i}$ and $0 \leq b \leq n_{j}$. Let $h$ be the first head in $R_{n_{j}, m, b}\left(D_{j}\right)$. Since $m \geq n_{j}$, the element $h$ exists and $h \leq n_{j}-1$. If $h$ is included in $R_{n_{i}, m, a}\left(D_{i}\right)$, we finish the proof. Otherwise, consider two elements $s$ and $t$ in $R_{n_{i}, m, a}\left(D_{i}\right)$ such that $s<h<t$.

Case 1: If $t \leq s+\phi$, we have $t \leq h+\phi-1 \leq$ $\left(n_{j}-1\right)+\phi-1$. Since $\left\lfloor\left(n_{i}-1\right) / 2\right\rfloor \geq 0$, we have $t \leq$ $m-1$. The element $t$ exists in $R_{n_{i}, m, a}\left(D_{i}\right)$. On the other hand, by definition of $D_{j}, h$ is a head of $R_{n_{j}, m, b}\left(D_{j}\right)$ implies that there exist $\phi-1$ continuous elements after $h$ in $R_{n_{j}, m, b}\left(D_{j}\right)$. The element $t$ must also be included in $R_{n_{j}, m, b}\left(D_{j}\right)$. We have $R_{n_{i}, m, a}\left(D_{i}\right) \cap R_{n_{j}, m, b}\left(D_{j}\right) \neq \varnothing$.

Case 2: If $t>s+\phi$, by definition of $D_{i}$, the element $t$ must be a head of $R_{n_{i}, m, a}\left(D_{i}\right)$. There exist $\phi-1$ continuous elements after $t$ in $R_{n_{i}, m, a}\left(D_{i}\right)$. Note that the element $t$ and all $\phi-1$ continuous elements after $t$ must be included in $R_{n_{i}, m, a}\left(D_{i}\right)$, since $d_{q_{i}-1} \geq\left(n_{i}-1\right) / 2$ implies that

$$
\begin{aligned}
t & \leq s+\left(n_{i}-\left(n_{i}-1\right) / 2\right)=s+\left(n_{i}+1\right) / 2 \\
& \leq h+\left(n_{i}+1\right) / 2-1=h+\left(n_{i}-1\right) / 2
\end{aligned}
$$

leading to $t \leq h+\left\lfloor\left(n_{i}-1\right) / 2\right\rfloor \leq\left\lfloor\left(n_{i}-1\right) / 2\right\rfloor+n_{j}-1=$ $m-\phi$, and therefore $t+\phi-1 \leq m-1$. On the other hand, all elements between $h$ and $t+\phi-1$ in $R_{n_{j}, m, b}\left(D_{j}\right)$ must have mutual distances less than or equal to $\phi$, since $h+d_{q_{j}-1} \geq$ $h+\left(n_{j}-1\right) / 2 \geq h+\left(n_{i}-1\right) / 2 \geq t$. By pigeon hole principle, there must exist at least one element between $t$ and $t+\phi-1$ in $R_{n_{j}, m, b}\left(D_{j}\right)$. Hence, $R_{n_{i}, m, a}\left(D_{i}\right) \cap R_{n_{j}, m, b}\left(D_{j}\right) \neq \varnothing$.

Comparison of the Two Schemes: As we can see, the EG and DS schemes generate a two-dimensional HQS in $O(1)$ time for any pair of stations. Both schemes are fully adaptive - stations can select any appropriate value of cycle length $\left(n_{i}\right)$ according to their own needs (e.g., delay requirements, remaining power, etc.) without losing the network connectivity.

However, stations adopting the EG and DS quorums may have distinct orientations in performance. Consider an example where $n$ varies from 4 to 20 . The buffering delay of $G_{20}$ and $D_{20}$ are four and nine beacon intervals, respectively. On the other hand, the quorum sizes of $G_{20}$ and $D_{20}$ are 8 and 6 , respectively. We can see that the EG scheme gives shorter buffering delay, therefore is more suitable for delay-constrained traffic; while the DS scheme yields smaller quorum size thus is more economic for best-effort traffic or circumstances when the battery power is critical.

It is important to note that stations in a network are free to switch between the EG and DS schemes.

Theorem 8: Given $d$ integers $n_{0}, n_{1}, \ldots, n_{d-1}$, where $n_{0}<$ $n_{1}<\cdots<n_{d-1}$. Let $\phi=\left\lceil\sqrt{\left(n_{d-1}+1\right) / 2}\right\rceil$. For any $i$ and $j, 0 \leq i, j \leq d-1$, the set $\left\{G_{i}, D_{j}\right\}$ forms an $\left(n_{i}, n_{j} ; n_{j}+\phi-\right.$ 1)-hyper quorum system.

Proof: See Appendix A.

The above theorem implies that any two stations using different schemes are guaranteed to discover each other within a bounded time. The interoperability between the EG and DS schemes offers extra flexibility to a QPS protocol in supporting both delay-constrained and best-effort traffic.

\section{HQS-Based Power Management}

In this section, we propose an HQS-based power-saving protocol, named HQPS, that gives enhanced scalability as compared to traditional QPS protocols. 


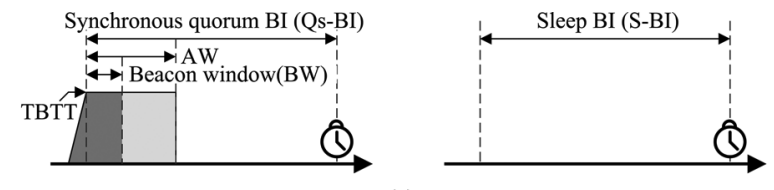

(a)

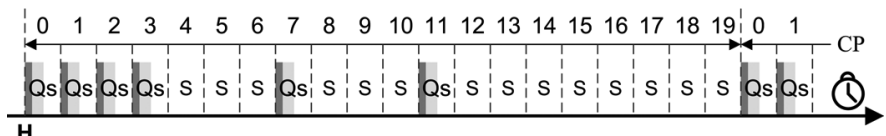

(b)
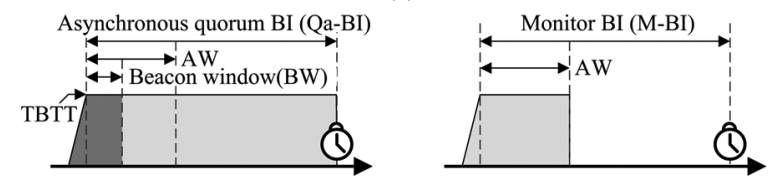

(c)

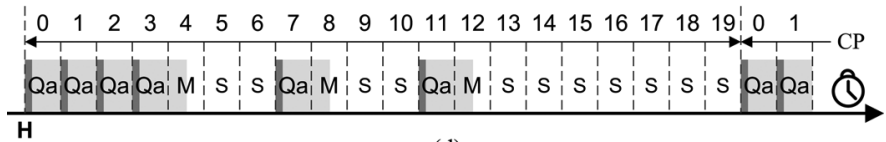

(d)

Fig. 3. Multimode time division in HQPS protocol. (a) Structural design of a beacon interval in synchronous mode. (b) Synchronous cycle pattern. (c) Structural design of a beacon interval in asynchronous mode. (d) Asynchronous cycle pattern.

Basically, the HQPS protocol inherits the design (e.g., beacon frames, neighbor maintenance, data transmission procedure, etc.) of a traditional QPS protocol. However, it defines two distinct cycle patterns for a quorum: the synchronous and asynchronous cycle patterns. Stations with the asynchronous cycle patterns, unlike those using the traditional (synchronous) cycle patterns as we have seen in Section II, have to remain awake during the entire beacon intervals whose numbers are specified in the quorums. They also need to remain awake during the first ATIM window of every series of sleep intervals. For example, consider a quorum $Q=\{0,1,2,3,7,11\}$. The structures of beacon intervals and their arrangement in a synchronous cycle pattern are shown in Fig. 3(a) and (b). This is what we have seen in Section II. Alternatively, a station may choose the asynchronous cycle pattern, as depicted in Fig. 3(c) and (d), if there is no TBTT alignment in the network.

Theorem 9: Any two stations $H_{0}$ and $H_{1}$ adopting the asynchronous cycle patterns for an $\left(n_{0}, n_{1} ; m\right)$-HQS are able to discover each other in $m+1$ beacon intervals without the need for TBTT alignment.

Proof: See Appendix B.

Generally, the synchronous cycle patterns result in better energy efficiency due to a smaller duty cycle; while the asynchronous cycle patterns render better scalability since no auxiliary timer synchronization mechanism is required. To combine the advantages of these two alternatives, the HQPS supports a hybrid topology, as illustrated in Fig. 4. Stations of the same region, e.g., Independent Basic Service Set (IBSS) [10] or cluster, where timer synchronization is cheap may pick the synchronous patterns to achieve better energy efficiency. Stations (gray nodes in Fig. 4) close to the border of two synchronized regions may adopt the asynchronous patterns to forward data. Note that given the same quorum, the neighbor discovery time of an asynchronous link (Fig. 4) is one beacon

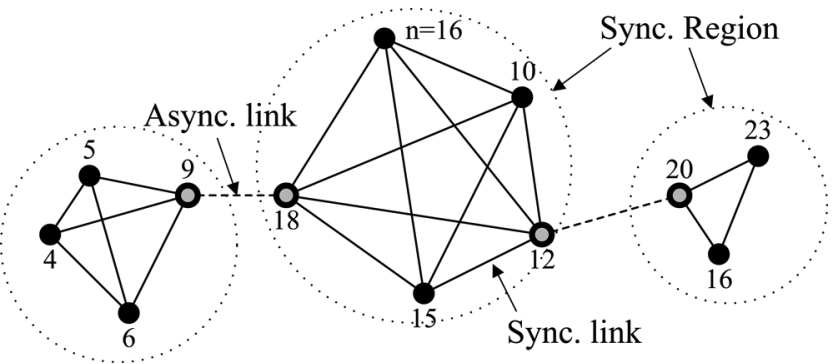

Fig. 4. Interoperability between the synchronous and asynchronous cycle patterns for large-scale environments where timer synchronization is difficult (or costly).

interval larger than that of the synchronous link; nevertheless, its buffering delay is one beacon interval smaller.

So far, we have explored a new quorum system and a multimode QPS protocol that offer each station a fine-tunable powersaving degree in either synchronous or asynchronous environments. However, two questions remain.

- In what circumstances shall a station switch from EG to DS scheme, and vice versa?

- What exact cycle length shall a station choose?

In previous studies [5], [7], these questions are answered empirically using the simulation results. In Section IV, we derive an analytic model that is able to determine a proper scheme and its optimal cycle length under a particular network condition.

\section{Optimal ADAPTATION}

In this section, we derive an analytic model that characterizes the performance of a QPS protocol. Stations can use this model to determine a proper scheme and its cycle length dynamically based on the current network condition and traffic loads. The model takes into account several performance requirements from the application or network layer, thereby enabling a cross-layer control of the adaptation.

Consider the two ends of a typical tradeoff between the performance and power saving.

1) How much performance degradation a station is willing to suffer (to increase the energy efficiency)?

2) How much benefit will a station earn from the increased energy efficiency (or accordingly, from the prolonged lifetime)?

Based on different application needs, the answers to the above questions may not be identical among stations. Our first task is to model these answers, which are only decidable at the network or application layer, as the input parameters. Notice that in a QPS protocol, the sending station can wake up and start the ATIM notification and data transmission procedures as soon as the receiver wakes up. The performance degradation is controlled by the wake up/sleep schedule of the receiving station. The first question can be answered by setting the parameters delay-threshold $h$ and drop-threshold $d$ for the receiving packets. These parameters indicate the maximally allowable delay and packet drop ratio when using a QPS protocol to receive data from neighbors. The answer to the second question can be set by another parameter called lifetime incentive, $e$. Let $E$ be the average energy consumption rate of a station, and $J$ be the total remaining power of that station. The term $J / E$ 


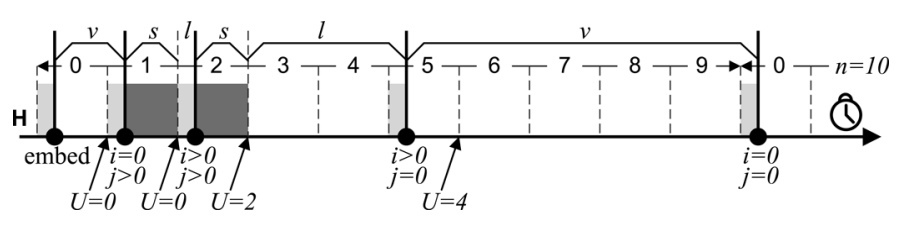

Fig. 5. Transitions between the embedded points (i.e., ends of the ATIM windows). The service process alternates between the serving (s), listening (l), and vacation (v) periods.

denotes the station's expected lifetime, and $(J / E)^{e}$ denotes the "worthiness" of that lifetime from the application's point of view. The larger the value of $e$, the more important a longer lifetime will be. For example, a station transmitting real-time data may indicate small thresholds; while a station running out of battery power may set large lifetime incentive. Given the above parameters and the current network condition, our next target is to find a proper quorum scheme and cycle length that strike the balance between the performance degradation and earned worthiness of the lifetime.

Based on the DCF models [4], [19] at the MAC layer, we perform our analysis at the packet level. We model the buffering queue of a sending station by using the semi-Markov process with states being the number of packets in queue. Consider a station $H$ that receives data from its neighbors. The packets arriving at a sending station must be buffered if $H$ is sleeping or in ATIM windows. When the number of buffered packets reaches the buffer capacity $K$, new arrival packets are blocked (i.e., dropped). Notice that the buffered packets can be transmitted only after the end of the next coming ATIM window on $H$ (where the ATIM notification procedure is performed, as described in Section II). Therefore, we embed the semi-Markov process to the ends of receiver $H$ 's ATIM windows, which are shown as the solid dots in Fig. 5, and study the state transition between these embedded points. By focusing on the wake up/sleep schedule of $H$, we are able to model the average energy consumption $E$ of $H$ as well as the drop ratio $D_{h}$ of packets destined to $H$.

The service process of this queue alternates between serving, listening, and vacation periods, as shown in Fig. 5. Packets can be served (i.e., transmitted) only during the serving periods. Denote $i$ and $j$ as the numbers of packets in queue at the previous and current embedded points, respectively. If $j>0$, the process enters the serving period and the data transmission starts. If $j=0$, the process enters the vacation period (as shown in the beacon intervals 5-9 and 0 in the next cycle in Fig. 5) to wait for the next embedded point. The length of a vacation period depends on the awake/sleep schedule guided by the adopted quorum and can be expressed as $(\bar{B}-\bar{A})+U \bar{B}+\bar{A}=(U+1) \bar{B}$, where $U$ is a random variable indicating how many beacon intervals a station can sleep after the current beacon interval by following the schedule. Note that after a serving period, there will not be an embedded point following up immediately due to the protocol design described in Section II. Hence, the process enters the listening period after each serving period until the coming of the next embedded point. The length of a listening period can be either $\bar{A}$ (e.g., in the beacon interval 2 in Fig. 5)
TABLE II

NotATION USED IN THE ANALYSIS

\begin{tabular}{|c|c|}
\hline$\overline{h, d, e}$ & Delay and drop thresholds, lifetime incentive. \\
\hline$n, \bar{A}, \bar{B}$ & $\begin{array}{l}\text { Cycle length, durations of the ATIM window and } \\
\text { beacon interval. }\end{array}$ \\
\hline$A_{x}, B_{x}$ & Transmission time of an ATIM frame and a beacon. \\
\hline$E_{x}, E_{i}, E_{s}$ & $\begin{array}{l}\text { Energy consumption rate of wireless module in } \\
\text { transmit/receive, idle, and sleep modes. }\end{array}$ \\
\hline$\lambda, \mu$ & Arrival and service rates. \\
\hline$K$ & Maximum queue size (in number of packets). \\
\hline$U$ & $\begin{array}{l}\text { Random variable indicating how many beacon } \\
\text { intervals a station may sleep after the current } \\
\text { beacon interval. }\end{array}$ \\
\hline$\pi_{i}$ & $\begin{array}{l}\text { Stationary probability of the queue having } i \text { packets } \\
\text { at the embedded point, } 1 \leq i \leq K \text {. }\end{array}$ \\
\hline$P_{a_{i}}$ & $\begin{array}{l}\text { Stationary probability of the queue in serving } \\
\text { periods starting with } i \text { packets. }\end{array}$ \\
\hline$P_{l_{i}}$ & $\begin{array}{l}\text { Stationary probability of the queue in listening } \\
\text { periods preceded by serving periods starting with } i \\
\text { packets. }\end{array}$ \\
\hline$P_{v}, P_{s}$ & $\begin{array}{l}\text { Stationary probabilities of the queue in vacation } \\
\text { periods and sleep mode. }\end{array}$ \\
\hline$P_{b}$ & Blocking probability. \\
\hline$\rho, \rho_{c}$ & Offered and carried load. \\
\hline$D_{h}$ & Delay drop ratio given the threshold $h$. \\
\hline$E$ & $\begin{array}{l}\text { Average energy consumption rate (in } \\
\text { transmitting/receiving } H \text { 's packets). }\end{array}$ \\
\hline
\end{tabular}

if the data transmission cannot finish within one beacon interval so the sender signals $H$ to remain awake in the next beacon interval, or $U \bar{B}+\bar{A}$ (e.g., in the beacon intervals 3-5 in Fig. 5) otherwise.

For ease of presentation, we assume the Poisson arrival pattern and exponential service time. ${ }^{5}$ The analysis can be readily extended to handle nonexponential service time by using the Markov ReGenerative Process (MRGP) [16]. We also assume that the nodes are synchronous in clocks. ${ }^{6}$ Table II summarizes the notation used in our analysis.

Note that the service rate $\mu$ equals the inverse of the effective channel rate determined by the DCF model, which covers the effects of random backoff, contention, channel interference, etc.

A quorum scheme and its cycle length affect the distribution of $U$. Consider a quorum $Q=\left\{q_{0}, q_{1}, \ldots, q_{|Q|-1}\right\} \subseteq$ $\{0,1, \ldots, n-1\}, U$ may be dependent with a particular cyclic set of $Q$ due to the clock shift on a station. However, since we are interested in the performance of a station with arbitrary clock shift, the $U$ can be modeled by the average of all distinct cyclic sets of $Q$. This implies that $U$ remains the same for every beacon interval on a station and can be expressed as

$$
U= \begin{cases}\frac{1}{n} \sum_{i=0}^{n-1}\left\{\left[\left(q_{j}-i\right) \bmod n\right]-1\right\}, & \text { if }|Q| \neq 1 \\ \frac{1}{n} \sum_{i=0}^{n-1}(n-i-1), & \text { otherwise }\end{cases}
$$

where $j, 0 \leq j \leq|Q|-1$, is the minimal index such that $\left(q_{j}-i\right) \bmod n \neq 0$ and $q_{j}$ is the "next" (cyclicly) element of $Q$ ahead the position $i$.

\footnotetext{
${ }^{5}$ Studies [25], [27] show that the discrepancy is not significant between the theoretical results derived under the Poisson assumption and the simulation results using the other packet arrival patterns.

${ }^{6}$ Analysis of the asynchronous condition is similar and therefore is omitted in this paper.
} 
Define a transition matrix $\boldsymbol{P}$ whose entry $\boldsymbol{P}_{i, j}$ denotes a conditional probability that there are $j$ packets in queue at the current embedded point (i.e., end of ATIM window) provided that there are $i$ packets in queue at the previous one. Observe that the queue may not reach a steady state between two embedded points, we characterize the transient behavior of the queue. By Chapman-Kolmogorov forward equation [8], $\boldsymbol{P}_{i, j}$ can be expressed as (1), shown at the bottom of the page, where $\boldsymbol{Q}$ is the corresponding $M / M / 1 / K$ generator matrix, by considering four different cases: 1) $i>0$ and $j\langle K$;2) $i>0$ and $j=K$; 3) $i=0$ and $j<K$; and 4) $i=0$ and $j=K$, respectively. In case 1 , we can see that the embedded point must be preceded by a serving period of length $\bar{B}-\bar{A}$, as $i>0$. If all packets are transmitted after the serving period, then the serving period is followed by a listening period of length $U \bar{B}+\bar{A}$ and there must be $j$ packets arriving during this listening period. Hence, the transition probability becomes

$$
\left[e^{\boldsymbol{Q}(\bar{B}-\bar{A})}\right]_{i, 0} \sum_{u=0}^{n-1} \frac{[\lambda(u \bar{B}+\bar{A})]^{j}}{j !} e^{-\lambda(u \bar{B}+\bar{A})} \operatorname{Pr}[U=u]
$$

by considering all possible values of $U$. Note that $\left[e^{\boldsymbol{Q}(\bar{B}-\bar{A})}\right]_{i, 0}$ is the transient probability [8] that the serving period transits from state $i$ to state 0 after a time interval of length $\bar{B}-\bar{A}$. Otherwise, suppose there are $s$ packets left at the end of the serving period, then the serving period is followed by a listening period of length $\bar{A}$ and there must be $j-s$ packets arriving during this listening period. The transition probability can be expressed as

$$
\sum_{s=1}^{j}\left[e^{\boldsymbol{Q}(\bar{B}-\bar{A})}\right]_{i, s} \frac{(\lambda \bar{A})^{(j-s)}}{(j-s) !} e^{-\lambda \bar{A}}
$$

by considering all possible values of $s$. The case 2 is similar to case 1 , except that there can be arbitrary numbers of packets larger than or equal to $K$ arriving at the listening period. In case 3 , we can see that the embedded point is preceded by a vacation period of length $(U+1) \bar{B}$, as $i=0$. There must be $j$ packets arriving during this period so the probability can be written as

$$
\sum_{u=0}^{n-1} \frac{(\lambda(u+1) \bar{B})^{j}}{j !} e^{-\lambda(u+1) \bar{B}} \operatorname{Pr}[U=u]
$$

by considering all possible values of $U$. The case 4 is similar to case 3 except that there can be arbitrary numbers of packets larger than or equal to $K$ arriving during the vacation period. The probability can be expressed as

$$
\sum_{u=0}^{n-1} \sum_{w=K}^{\infty} \frac{(\lambda(u+1) \bar{B})^{w}}{w !} e^{-\lambda(u+1) \bar{B}} \operatorname{Pr}[U=u] .
$$

Let $\boldsymbol{\pi}=\left[\pi_{0}, \pi_{1}, \ldots, \pi_{K}\right]$, we may obtain $\pi_{i}$ by solving the Chapman-Kolmogorov equation [20], $\boldsymbol{\pi} \boldsymbol{P}=\boldsymbol{\pi}$, and $\sum_{i=0}^{K} \pi_{i}=$ 1. Note that by definition, $P_{a_{i}}, P_{l_{i}}$, and $P_{v}$ are equal to the portion of time the queue remains in serving, listening, and vacation periods, respectively, we have $P_{a_{i}}=\pi_{i}(\bar{B}-\bar{A}) / \Omega, P_{l_{i}}=$ $\pi_{i}\left(\left[e^{\boldsymbol{Q}(\bar{B}-\bar{A})}\right]_{i, 0} E[U] \bar{B}+\bar{A}\right) / \Omega$, and $P_{v}=\pi_{0}(E[U]+1) \bar{B} / \Omega$, where $\Omega$ given by

$$
\begin{aligned}
\Omega= & \pi_{0}(E[U]+1) \bar{B} \\
& +\sum_{i=1}^{K} \pi_{i}\left\{(\bar{B}-\bar{A})+\left[e^{\boldsymbol{Q}(\bar{B}-\bar{A})}\right]_{i, 0} E[U] \bar{B}+\bar{A}\right\}
\end{aligned}
$$

is a normalization term ensuring that $P_{a_{i}}, P_{l_{i}}$, and $P_{v}$ are less than 1 . In addition

$$
P_{s}=\frac{\pi_{0}\{(E[U]+1) \bar{B}-\bar{A}\}+\sum_{i=1}^{K} \pi_{i}\left[e^{\boldsymbol{Q}(\bar{B}-\bar{A})}\right]_{i, 0} E[U] \bar{B}}{\Omega} .
$$

To determine $E$, we need to derive $P_{b}$ first. Consider an arriving packet that may fall into the queue during the serving, listening, or vacation periods. Let $\left.P_{b}\right|_{a_{i}}$ be the blocking probability conditioned on the fact that the packet comes during a serving period starting from $i$ packets. From PASTA [23], $\left.P_{b}\right|_{a_{i}}$ is equal to the probability that the arriving packet sees $K$ packets in queue. Note that Poisson arrivals can be thought of a random point process on the time axis, we have

$$
\left.P_{b}\right|_{a_{i}}=\frac{1}{\bar{B}-\bar{A}} \int_{t=0}^{\bar{B}-\bar{A}}\left[e^{\boldsymbol{Q} t}\right]_{i, K} d t .
$$

Applying the similar analysis, we may obtain the conditional blocking probabilities, $\left.P_{b}\right|_{l_{i}}$ and $\left.P_{b}\right|_{v}$, for the listening and vacation periods, respectively. Note that the packet comes during these periods must be buffered, therefore, we have $\left.P_{b}\right|_{l_{i}}$

$$
P_{i, j}=\left\{\begin{array}{lr}
{\left[e^{\boldsymbol{Q}(\bar{B}-\bar{A})}\right]_{i, 0} \sum_{u=0}^{n-1} \frac{[\lambda(u \bar{B}+\bar{A})]^{j}}{j !} e^{-\lambda(u \bar{B}+\bar{A})} \operatorname{Pr}[U=u]+\sum_{s=1}^{j}\left[e^{\boldsymbol{Q}(\bar{B}-\bar{A})}\right]_{i, s} \frac{(\lambda \bar{A})^{(j-s)}}{(j-s) !} e^{-\lambda \bar{A}},} & \text { if } i>0, j<K \\
{\left[e^{\boldsymbol{Q}(\bar{B}-\bar{A})}\right]_{i, 0} \sum_{u=0}^{n-1} \sum_{w=K}^{\infty} \frac{[\lambda(u \bar{B}+\bar{A})]^{w}}{w !} e^{-\lambda(u \bar{B}+\bar{A})} \operatorname{Pr}[U=u]+\sum_{s=0}^{K}\left[e^{\boldsymbol{Q}(\bar{B}-\bar{A})}\right]_{i, s} \sum_{w=K-s}^{\infty} \frac{(\lambda \bar{A})^{w}}{w !} e^{-\lambda \bar{A}},} & \text { if } i>0, j=K \\
\sum_{u=0}^{n-1} \frac{(\lambda(u+1) \bar{B})^{j}}{j !} e^{-\lambda(u+1) \bar{B}} \operatorname{Pr}[U=u], & \text { if } i=0, j<K \\
\sum_{u=0}^{n-1} \sum_{w=K}^{\infty} \frac{(\lambda(u+1) \bar{B})^{w}}{w !} e^{-\lambda(u+1) \bar{B}} \operatorname{Pr}[U=u], & \text { if } i=0, j=K
\end{array}\right.
$$


as (3), shown at the bottom of the page. In (3), if all packets can be transmitted within the preceding serving period, then the blocking probability equals to the probability that the arriving packet sees $K$ packets in the buffer at some time $t$ within the following listening period of length $U \bar{B}+\bar{A}$, leading to the first term. Otherwise if there are $s$ packets remaining after the end of the serving period, the blocking probability equals to the probability that the arriving packet sees $K$ packets at some time $t$ within the following listening period of length $\bar{A}$, leading to the second term. Similarly

$\left.P_{b}\right|_{v}=\sum_{u=0}^{n-1}\left\{\frac{1}{(u+1) \bar{B}} \int_{t=0}^{(u+1) \bar{B}} \sum_{w=K}^{\infty} \frac{(\lambda t)^{w}}{w !} e^{-\lambda t} d t\right\}$ $\cdot \operatorname{Pr}[U=u]$.

Putting $\left.P_{b}\right|_{a_{i}},\left.P_{b}\right|_{l_{i}}$, and $\left.P_{b}\right|_{v}$ together, we obtain the unconditional blocking probability

$$
P_{b}=\sum_{i=1}^{K}\left(\left.P_{b}\right|_{a_{i}} P_{a_{i}}+\left.P_{b}\right|_{l_{i}} P_{l_{i}}\right)+\left.P_{b}\right|_{v} P_{v} .
$$

Given $\rho=\lambda / \mu$, the carried load can be expressed as $\rho_{c}=$ $\left(1-P_{b}\right) \rho$. This leads to

$$
E=E_{x}\left(\rho_{c}+P_{A B}\right)+E_{s} P_{s}+E_{i}\left(1-\rho_{c}-P_{A B}-P_{s}\right)
$$

where

$$
P_{A B}=\sum_{i=1}^{K} \frac{A_{x}+B_{x}}{\left[e^{\boldsymbol{Q}(\bar{B}-\bar{A})}\right]_{i, 0} E[U] \bar{B}+\bar{A}} P_{l_{i}}+\frac{B_{x}}{(E[U]+1) \bar{B}} P_{v}
$$

denotes the stationary probability that the sending station transmits the ATIM and beacon frames.

Next, we derive $D_{h}$, which, by definition, equals the probability that the buffering and transmission delay of a packet is longer than $h$. Let $D$ be a random variable denoting the delay encountered by the packet, we have $D_{h}=1-\operatorname{Pr}[D \leq h]$. Consider the FIFO service discipline, an arriving packet has to wait until all packets buffered ahead are served. If the packet arrives during the serving periods starting from $i$ packets, we have

$$
\left.D_{h}\right|_{a_{i}}=1-\frac{1}{\bar{B}-\bar{A}} \int_{t=0}^{\bar{B}-\bar{A}} \sum_{j=0}^{K-1}\left[e^{\boldsymbol{Q} t}\right]_{i, j} E r_{j+1, h} d t
$$

where

$$
E r_{j+1, h}=1-\sum_{s=0}^{j} \frac{(\mu h)^{s}}{s !} e^{-\mu h}
$$

is the cumulative distribution function (CDF) of Erlang distribution denoting the probability that the sum of service time of $j-1$ preceding packets, residual time of the $j$ th preceding packet, and service time of current arrival is less than or equal to $h$. If the packet arrives during the listening or vacation periods, it has to wait until the end of the current period and service completion of all the packets buffered ahead. Therefore, $\left.D_{h}\right|_{l_{i}}$ and $\left.D_{h}\right|_{v}$ can be expressed as (4) and (5), respectively, shown at the bottom of the page. In (4), if all packets are transmitted within the preceding serving period, then $P[D \leq h]$ equals to the probability that a new packet arriving at time $t$ within the listening period given that there are already $j$ packets arrived in the same period can be served before the deadline $h-(U \bar{B}+\bar{A}-t)$ in the later serving periods, leading to the second term. Otherwise, $P[D \leq h]$ equals to the probability that a new packet arriving at time $t$ within the listening period given that there are already $j$ packets arrived in the same period and $s$ additional packets remaining after the preceding serving period can be served before the deadline $h-(\bar{A}-t)$ in the later serving periods, leading to the third term. Equation (5) can be explained similarly.

The unconditional drop ratio is given by

$$
D_{h}=\sum_{i=1}^{K}\left(\left.D_{h}\right|_{a_{i}} P_{a_{i}}+\left.D_{h}\right|_{l_{i}} P_{l_{i}}\right)+D_{h} \mid{ }_{v} P_{v} .
$$

\section{A. Optimal Adaptation}

A good power management protocol should increase the lifetime value $(J / E)^{e}$ of a station without causing intolerable

$$
\begin{aligned}
\left.P_{b}\right|_{I_{i}}= & {\left[e^{\boldsymbol{Q}(\bar{B}-\bar{A})}\right]_{i, 0} \sum_{u=0}^{n-1}\left\{\frac{1}{u \bar{B}+\bar{A}} \int_{t=0}^{u \bar{B}+\bar{A}} \sum_{w=K}^{\infty} \frac{(\lambda t)^{w}}{w !} e^{-\lambda t} d t\right\} \operatorname{Pr}[U=u]+\sum_{s=1}^{K}\left[e^{\boldsymbol{Q}(\bar{B}-\bar{A})}\right]_{i, s}\left\{\frac{1}{\bar{A}} \int_{t=0}^{\bar{A}} \sum_{w=K-s}^{\infty} \frac{(\lambda t)^{w}}{w !} e^{-\lambda t} d t\right\} } \\
\left.D_{h}\right|_{l_{i}}= & 1-\left[e^{\boldsymbol{Q}(\bar{B}-\bar{A})}\right]_{i, 0} \sum_{u=0}^{n-1}\left\{\frac{1}{u \bar{B}+\bar{A}} \int_{t=0}^{u \bar{B}+\bar{A}} \sum_{j=0}^{K-1} \frac{(\lambda t)^{j}}{j !} e^{-\lambda t} \operatorname{Er} r_{j+1, h-(u \bar{B}+\bar{A}-t)} d t\right\} \operatorname{Pr}[U=u] \\
& -\sum_{s=1}^{K-1}\left[e^{\boldsymbol{Q}(\bar{B}-\bar{A})}\right]_{i, s}\left\{\frac{1}{\bar{A}} \int_{t=0}^{\bar{A}} \sum_{j=0}^{K-1-s} \frac{(\lambda t)^{j}}{j !} e^{-\lambda t} \operatorname{Er}_{s+j+1, h-(\bar{A}-t)} d t\right\} \\
\left.D_{h}\right|_{v}= & 1-\sum_{u=0}^{n-1}\left\{\frac{1}{(u+1) \bar{B}} \int_{t=0}^{(u+1) \bar{B}} \sum_{j=0}^{K-1} \frac{(\lambda t)^{j}}{j !} e^{-\lambda t} \operatorname{Er}_{j+1, h-((u+1) \bar{B}-t)} d t\right\} \operatorname{Pr}[U=u] .
\end{aligned}
$$




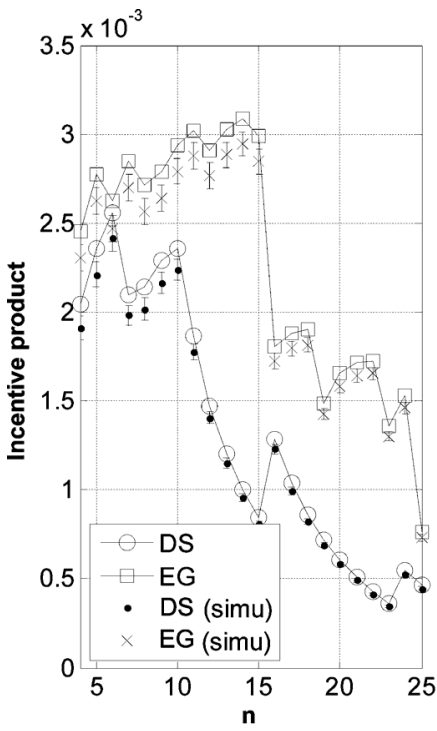

(a)

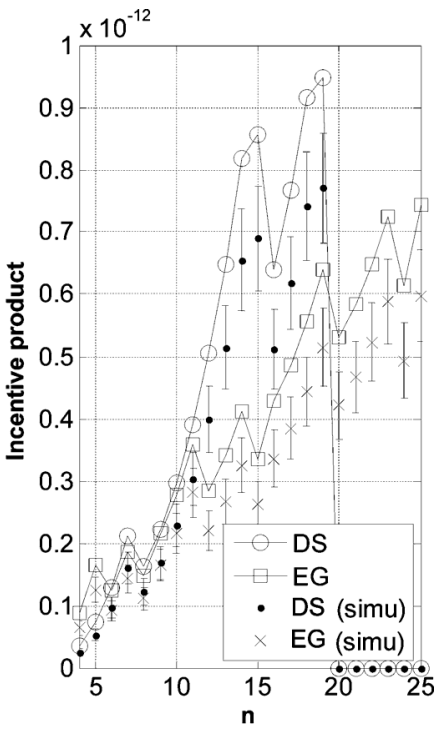

(b)
Fig. 6. Incentive products returned by EG and DS schemes given (a) delayconstrained traffic $(h=300 \mathrm{~ms}, d=0.2)$ and high battery power $(e=1)$ and (b) best-effort traffic ( $h=0.8 \mathrm{~s}, d=0.01)$ and low battery power $(e=5)$.

performance degradation. Following the previous analysis, we obtain a normalized performance drop ratio $D_{h} / d$ in terms of the thresholds $h$ and $d$. To obtain a proper configuration, a station may pick a quorum scheme and cycle length that together maximize the incentive product, i.e.,

$$
\left(1-\frac{D_{h}}{d}\right) \cdot\left(\frac{1}{E}\right)^{e}
$$

between itself and every nearby sender. Note that the $(1 / E)^{e}$ term corresponds to the lifetime value $(J / E)^{e}$. Generally, when the cycle length $n$ is increased, the incentive product raises correspondingly because of $(1 / E)^{e}$. The lifetime value $(1 / E)^{e}$ grows as the duty cycle is reduced. However, when $n$ becomes too large, the incentive product drops due to the intolerable performance degradation reflected by $\left(1-D_{h} / d\right)$.

Although the above tendency holds generally, different schemes may result in different curves. Fig. 6 shows typical outputs of the EG and DS schemes (given parameters $\rho=0.02$, $K=64, \bar{A}=25 \mathrm{~ms}$, and $\bar{B}=100 \mathrm{~ms}$ ). As we can see in Fig. 6(a), when a station is transmitting the delay-constrained traffic (e.g., voice/video packets with a tight delay bound $h=300 \mathrm{~ms}$ [9] and tolerable loss rate $d=0.2$ [18]) or when it has high battery power (which implies a low lifetime incentive $e=1$ ), the EG scheme gives better incentive products. In particular, it reaches the optimum at $n=14$. On the other hand, when transmitting the best-effort traffic (with a loose delay bound $h=0.8 \mathrm{~s}$ slightly lower than the route discovery timeout, $1 \mathrm{~s}$ [21], but strict loss rate $d=0.01$ avoiding retransmissions) or when the battery power is critical $(e=5)$, the DS scheme returns better incentive products. In this case, $n=19$ reaches the optimum. Note that the maximal cycle length may be limited by the node mobility or the route advertisement interval at the network layer. In this paper, we consider $n \leq 25$ to assure in-time neighbor discovery/maintenance. By precalculating all incentive products with different input parameters, each station can look up the optimal scheme and cycle length in $O(1)$ time.

Note that this model is validated by extensive simulations. We include the simulation results in Fig. 6 with 95\% confidence intervals using default parameters that will be described in Section V. The error comes from the fact that we ignore the energy consumption in transmitting beacon and ATIM frames in analytic plots. Notice that this error may be magnified when we choose a large $e$ due to its nonlinearity. Nevertheless, the tendency of each simulation curve can still be captured successfully.

\section{Performance Evaluation}

In this section, we evaluate the performance of HQPS by taking both the theoretical analysis and simulation results. We implement our simulation based on the $n s-2$ simulator [1] with CMU wireless extension. The simulation is conducted in a $500 \times 500-\mathrm{m}^{2}$ static network with 50 randomly distributed stations. Each station has half-duplex wireless channel of rate $2 \mathrm{Mb} / \mathrm{s}$ and transmission range $100 \mathrm{~m}$. We set the duration of beacon interval and ATIM window to 100 and $25 \mathrm{~ms}$, respectively. The mean packet size is 256 , and each station is supplied with the Poisson traffic [25] with rate varying from 2 to $t_{\max } \mathrm{kb} / \mathrm{s}$, where $t_{\max }=40$ by default. The power consumption rates of the wireless module are set 1650, 1400, 1150 , and $45 \mathrm{~mW}$ in transmit, receive, idle, and sleep modes, respectively . For simplicity, we focus on the situation that all stations are synchronous in their clocks. The benefit offered by HQPS in asynchronous environments is analogous to that of synchronous environments since, given the same quorum scheme, stations in these two environments differ only in the structure of beacon intervals (as we can see in Fig. 3), rather than the portion of awake beacon intervals per cycle.

To evaluate the performance of a quorum scheme, we define a theoretical metric-quorum ratio, which denotes the proportion of beacon intervals in a cycle where a station is required to awake. Specifically, it is defined as $|Q| / n$, where $|Q|$ is the quorum size and $n$ is the cycle length. ${ }^{7}$

We compare HQPS to the previous studies AQEC [5] and AAPM [7]. These two protocols adopt Grid and FPP as the underlying quorum schemes, respectively. To ensure valid neighbor maintenance in routing protocols [21], the cycle length $n$ is considered to be less than or equal to $n_{\max }=25$. We set $\phi=4$. Both AQEC and AAPM adopt the rule-based

\footnotetext{
${ }^{7}$ Note that, traditionally, the performance of a quorum system can be measured by another metric: the number of common elements between a pair of quorums. However, in a QPS protocol, this number is a less significant factor to the energy efficiency than the quorum ratio. Consider two pairs of quorums $\left(Q_{1}, Q_{2}\right)$ and $\left(Q_{3}, Q_{4}\right)$. Suppose all these quorums give the same quorum ratio, but $\left|Q_{1} \cap Q_{2}\right|=1$ and $\left|Q_{3} \cap Q_{4}\right|=2$. If there is no data transmission, then all these four quorums yield the same level of energy efficiency. On the other hand, when transmitting data, a sender node can wake up actively whenever the next ATIM window comes on the receiving station, making the extra intersection be of little help on energy saving (nor on buffering delay since it is the "distances" between elements that matter). The energy efficiency on respective stations choosing $Q_{1}, Q_{2}, Q_{3}$, and $Q_{4}$ will still be similar given the same traffic load. Therefore, we do not consider this metric in our evaluation.
} 
adaptation criteria [5] to dynamically adjust $n$. In our experiments, the rules for AQEC are

\begin{tabular}{cc} 
Grid size $(n)$ & Used when \\
\hline $1 \times 1(1)$ & traffic $\geq t_{\max } \cdot(1 / 1)$ \\
$2 \times 2(4)$ & $t_{\max } \cdot(1 / 1)>\operatorname{traffic} \geq t_{\max } \cdot(3 / 4)$ \\
$3 \times 3(9)$ & $t_{\max } \cdot(3 / 4)>$ traffic $\geq t_{\max } \cdot(5 / 9)$ \\
$4 \times 4(16)$ & $t_{\max } \cdot(5 / 9)>$ traffic $\geq t_{\max } \cdot(7 / 16)$ \\
$5 \times 5(25)$ & $t_{\max } \cdot(7 / 16)>$ traffic $\geq t_{\max } \cdot(9 / 25)$ \\
\hline
\end{tabular}

Similarly, AAPM uses the rules corresponding to the quorum ratios offered by FPP. On the other hand, HQPS follows the analytic model given in Section IV to pick a proper scheme and $n$. With this model, HQPS may have different behavior when different prerequisites (i.e., $h, d$, and $e$ ) are given. We consider two typical scenarios where delay-constrained and best-effort packets are offered. In the former case, we set $h=300 \mathrm{~ms}$, $d=0.2$, and $e=1$; in the latter, we set $h=0.8 \mathrm{~s}, d=0.01$, and $e=5$. We denote the HQPS with these two prerequisite settings HQPS(D) and HQPS(B), respectively. Note that to give a fair comparison, all protocols employ the same design of cycle pattern as given in Fig. 3(b).

\section{A. Theoretical Analysis: The Quorum Ratio}

We first explore the quorum ratio of different schemes by varying the cycle length $n$ from 1 to $n_{\max }$. As shown in Fig. 7(a), all schemes give smaller quorum ratios as $n$ increases. In particular, the ratio of FPP approaches the theoretical bound $1 / \sqrt{n}$ [12]. This ratio is shown to be optimal [7]. However, the optimum in quorum size does not necessarily imply the optimum in performance. Notice that FPP and Grid cannot produce quorums given arbitrary cycle lengths. This leads to a sparse configuration density. During the rule-based adaptation, $n$ may shrink very fast when there is only little increment in traffic load. A station may tend to remain awake more than necessary. Note that the quorum ratios of Grid and EG overlap when $n$ is a square. This is simply because EG is a generalization of the Grid scheme.

Next, we evaluate the performance of different protocols based on the simulation results.

\section{B. Link Discovery Time}

In this section, we study the link discovery time. As illustrated in Fig. 7(b), all protocols are able to discover more than $90 \%$ of the links within 2 seconds. Interestingly, although in theory the worst-case neighbor discovery time should be larger than $n_{\max } \bar{B}=2.5$ seconds (for example, two stations $H_{0}$ and $H_{1}$ adopting $n_{0}=16$ and $n_{1}=25$ respectively and DS scheme may have the worst-case neighbor discovery time $\left(\left\lfloor\sqrt{n_{0}}\right\rfloor+n_{1}-1\right) \bar{B}=2.8$ seconds), AQEC and HQPS achieve $100 \%$ link discovery ratio at 1.5 and 2.5 seconds respectively. In practice, AQEC and HQPS work quite well. We notice that AAPM occasionally gives long neighbor discovery time since FPP does not guarantee a theoretical bound of the worst-case neighbor discovery time. We cannot ensure $100 \%$ link discovery by limiting the value of $n$.

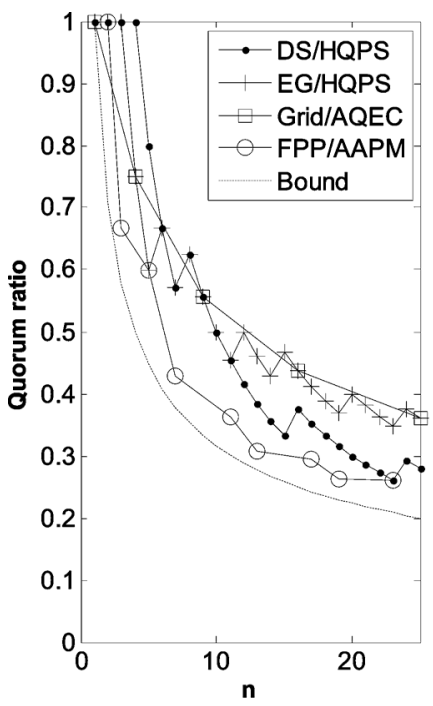

(a)

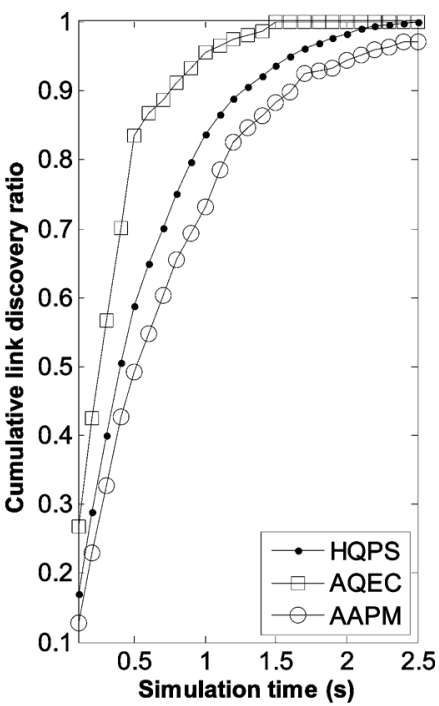

(b)
Fig. 7. (a) Quorum ratio and (b) average neighbor discovery time.

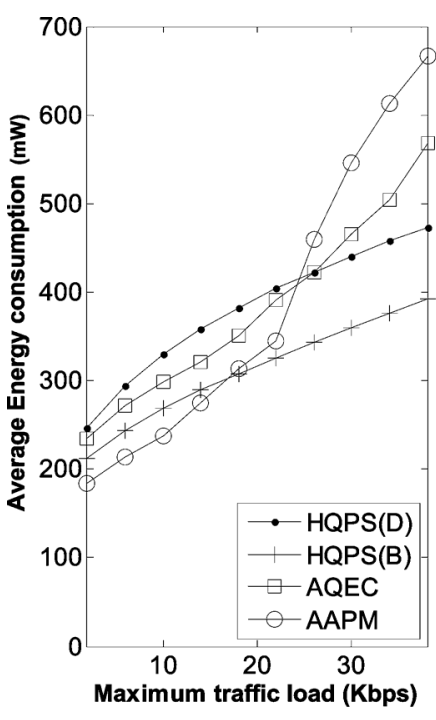

(a)

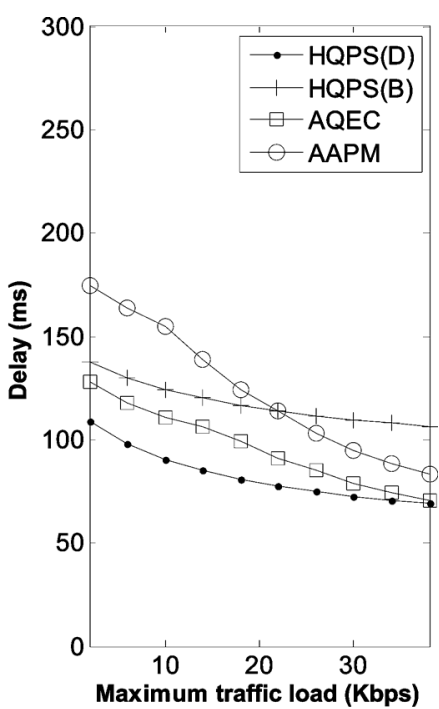

(b)
Fig. 8. (a) Average energy consumption rate and (b) delay.

\section{Energy Conservation}

In this section, we evaluate the energy efficiency of all protocols under different loads. We vary $f_{\max }$ from 2 to $40 \mathrm{~kb} / \mathrm{s}$. The experimental results are shown in Fig. 8(a). As we can see, all protocols give higher energy consumption rate as $f_{\max }$ increases. This is because of the frequent ATIM notification and data transmission procedures. Notice that the energy consumption rates of AQEC and AAPM grow significantly when $f_{\max }>20 \mathrm{~kb} / \mathrm{s}$. This is mainly due to the sparse configuration density, as we have seen in Fig. 7(a). When the offered load is close to the maximum support load, $n$ shrinks very fast and keeps a station awake most of time. On the other hand, HQPS gives relatively stable energy consumption rate under all loads. As compared to AQEC and AAPM, HQPS offers competitive 


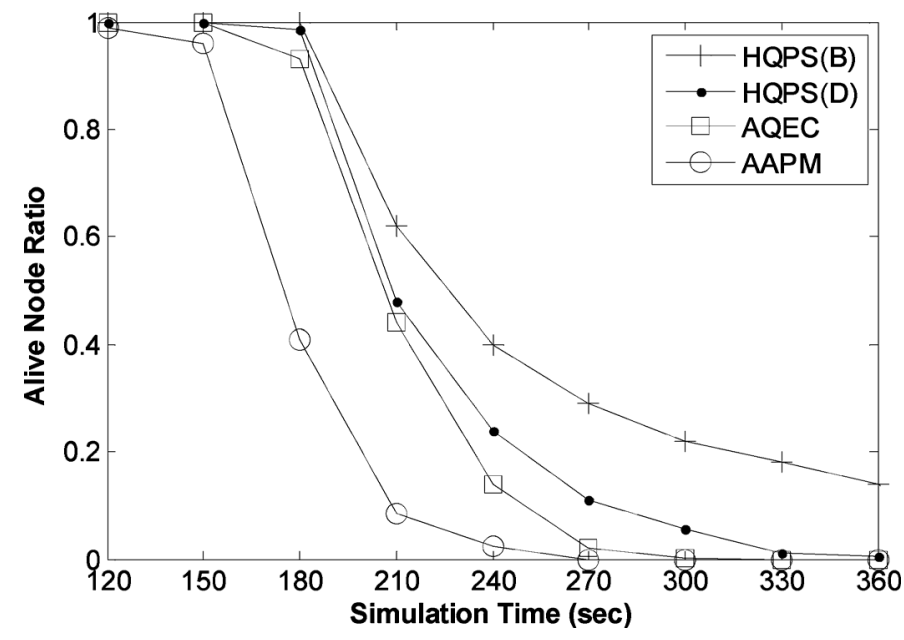

Fig. 9. Ratio of alive nodes.

energy efficiency under light traffic loads while giving respectively up to $31 \%$ and $41 \%$ reduction in energy consumption rate under heavy loads.

Next, we look further into the energy efficiency by studying the lifetime of nodes in a network. In this experiment, we set $f_{\max }=30 \mathrm{~kb} / \mathrm{s}$, give initial energy $100 \mathrm{~J}$ to each node, and record the ratio of alive nodes every $30 \mathrm{~s}$ of the simulation time. The results are shown in Fig. 9. As we can see, both the HQPS(D) and HQPS(B) improve the network lifetime when compared to AQEC and AAPM. The improvement mainly comes from the HQPS(D)'s and HQPS(B)'s "longer tails" in the zone of low alive node ratio (below 0.5 ). We believe these tails are the results of the finer adaptation granularity offered by the EG and DS schemes. When the number of alive nodes is high, each node receives packets from nearby nodes, and the aggregated receiving traffic will be uniformly high among the nodes. The adaptation strategies force all protocols to choose a small cycle length, so the advantages of EG and DS are not obvious. However, when the number of alive nodes decreases, the receiving traffic becomes diverse between nodes. With the finer adaptation granularity offered by the EG and DS schemes, these nodes can have a higher chance to find quorums that achieve high energy efficiency without causing unacceptable performance degradation. Therefore, the nodes with light traffic load can survive and form a longer tail. Note that as compared to HQPS(D), HQPS(B) tends to find quorums that lead to higher energy efficiency, so HQPS(B) gives even longer tails than HQPS(D) does. Also notice that in AAPM, the number of alive nodes starts to fall earlier than other protocols. We believe this is because of the the sparse configuration density offered by the FPP scheme at small $n$ 's.

\section{Delay and Delay Drop Ratio}

In this section, we first investigate the average delay encountered during each packet transmission. As we can see in Fig. 8(b), the delay in all protocols decreases as the load becomes heavier. This is because, under high loads, a station remains awake most of time, thereby reducing the data buffering delay. Note that HQPS may give different performance orientations when different prerequisites (i.e., $h, d$, and $e$ ) are

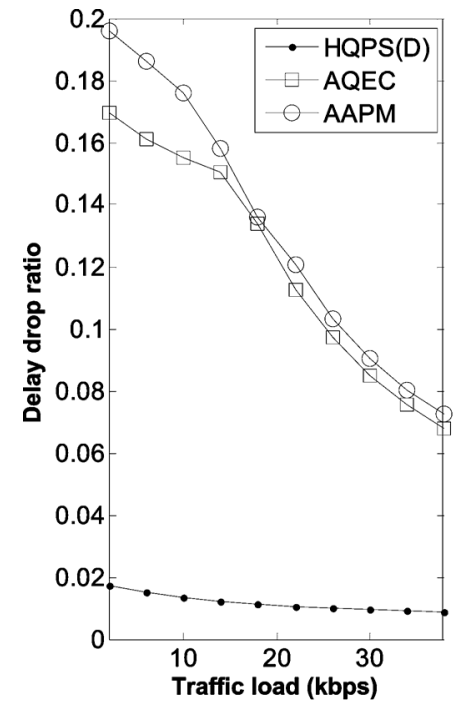

(a)

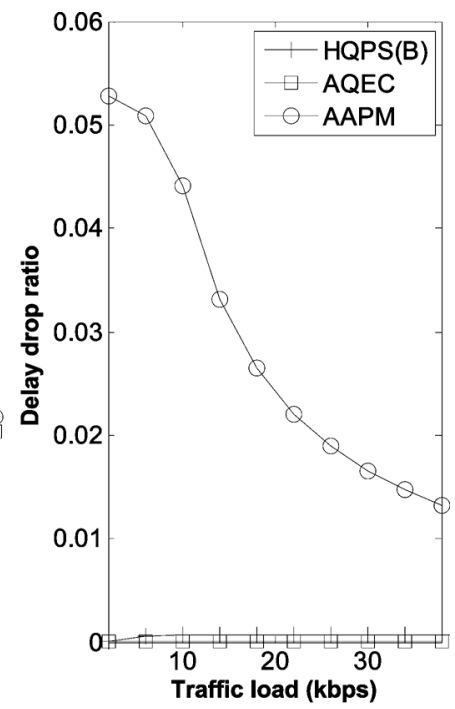

(b)
Fig. 10. Delay drop ratio given (a) delay-constrained traffic and (b) best-effort traffic.

provided. For best-effort traffic, HQPS(B) gives higher delay in exchange for better energy efficiency. On the other hand, for delay-constrained traffic, the delay is reduced in HQPS(D) at the cost of extra energy consumption.

It is important to realize that the power-saving effect of HQPS comes with a performance guarantee. In HQPS, energy is saved only when there is no intolerable performance degradation. As shown in Fig. 10, AQEC and AAPM suffer from serious delay drops under light loads. Many studies [2], [14], [28] show that the cost of these drops may easily drown the benefit in energy saving. Our model compromises these to conflict goals when adapting $n$. The results show that under all loads, either HQPS(D) or HQPS(B) is able to suppress more than $90 \%$ of the drops for both the delay-constrained and best-effort traffic.

\section{CONCLUSION}

In this paper, we generalized traditional quorum systems and proposed the Hyper Quorum System. We showed that HQS is fully adaptive in the sense that a station can select any value of cycle length that is best suited to its own requirements in terms of packet delay and power constraint. Two HQS constructing schemes were presented that facilitate power saving under both delay-constrained and best-effort traffic. Both the constructing schemes run in $O(1)$ time, which may be readily adopted by nodes (e.g., sensors) with limited computing power. We derived an analytical model characterizing the performance of a QPS protocol, based on which stations can determine a proper scheme and the optimal cycle length. Experimental results showed that our HQS-based power management protocol renders significantly more stable performance under various traffic loads as compared to traditional QPS protocols. In particular, it gives up to $41 \%$ improvement in energy efficiency under heavy traffic loads while eliminating more than $90 \%$ delay drops under light traffic loads.

The model accepts prerequisite parameters $h, d$, and $e$. The best values of these parameters cannot be determined at the 


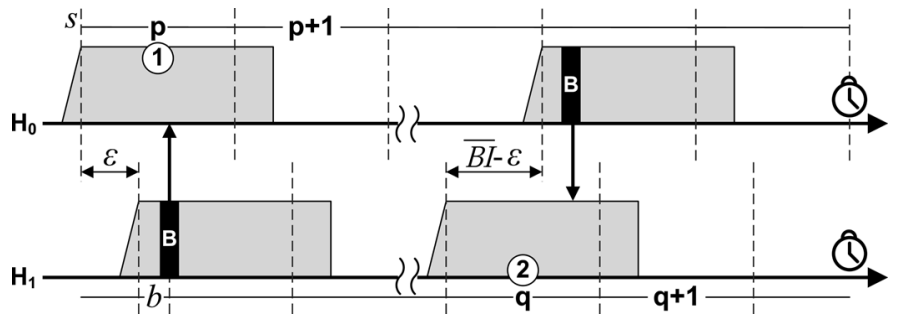

Fig. 11. Guaranteed neighbor discovery with the asynchronous cycle patterns.

MAC layer alone, as it involves Network- and Applicationlayer considerations (e.g., routing advertisement period, network topology, application state, user preference, etc.). A crosslayer technique for choosing the parameters would be valuable and is left as a future work.

\section{APPENDIX A \\ PROOF OF THEOREM 8}

Let $m=n_{j}+\phi-1$, we show that $R_{n_{i}, m, a}\left(G_{i}\right) \cap$ $R_{n_{j}, m, b}\left(D_{j}\right) \neq \varnothing$ for all $a$ and $b, 0 \leq a \leq n_{i}$ and $0 \leq b \leq n_{j}$. Recall that the heads of $R_{n_{j}, m, b}\left(D_{j}\right)$ are the elements projected from the first element 0 in $D_{j}$. Let $h$ be the first head in $R_{n_{j}, m, b}\left(D_{j}\right)$. Since $m \geq n_{j}, h$ exists and $h \leq n_{j}-1$. If $h$ is included in $R_{n_{i}, m, a}\left(G_{i}\right)$, we finish the proof. Otherwise, consider two elements $s$ and $t$ in $R_{n_{i}, m, a}\left(G_{i}\right)$ such that $s<h<t$. By definition of $G_{i}$, any two interspaced elements in $R_{n_{i}, m, a}\left(G_{i}\right)$ must have mutual distance less than or equal to $\phi_{i}$, which is less than or equal to $\phi$. We have $t \leq s+\phi_{i} \leq s+\phi$, leading to $t \leq h+\phi-1 \leq\left(n_{j}-1\right)+\phi-1=m-1$. The element $t$ exists in $R_{n_{i}, m, a}\left(G_{i}\right)$. On the other hand, by definition of $D_{j}, h$ is a head of $R_{n_{j}, m, b}\left(D_{j}\right)$ implies that there exist $\phi-1$ continuous elements after $h$ in $R_{n_{j}, m, b}\left(D_{j}\right)$. Since $t \leq h+\phi-1$, the element $t$ must also be included in $R_{n_{j}, m, b}\left(D_{j}\right)$. We have $R_{n_{i}, m, a}\left(G_{i}\right) \cap R_{n_{j}, m, b}\left(D_{j}\right) \neq \varnothing$.

\section{APPENDIX B}

\section{ProOF OF THEOREM 9}

Consider two stations, $H_{0}$ and $H_{1}$. Let $\epsilon, 0 \leq \epsilon \leq \bar{B}$, denote the TBTT shift between these two hosts, as illustrated in Fig. 11. We first show that $H_{0}$ is able to receive $H_{1}$ 's beacons within a finite amount of time. If $\epsilon=0$, from Theorems 6 and 7 we know there must exist a Qa-BI [see Fig. 3(c)], named $q$, on $H_{0}$ that intersects with a Qa-BI on $H_{1}$ [Fig. 11 (1)]. $H_{0}$ is able to receive $H_{1}$ 's beacon during the beacon window of $p$. If $\epsilon>0$, let $s$ denote the starting time of $p$, and let $b$ denote the elapsed time between $s+\epsilon$ and beacon reception. Note that $0 \leq b \leq \bar{W}$, where $\bar{W}$ is the duration of a beacon window. As we can see, $H_{1}$ 's beacon arrives at time $s+b+\epsilon$. Since $\epsilon \leq \bar{B}$, the arrival must fall within the time range $(s+b, s+b+\bar{B}]$. Observe that in an asynchronous cycle pattern, each Qa-BI can only be followed by either the Qa-BI or M-BI. By definition of the asynchronous cycle pattern, $H_{0}$ must remain active during the time range $[s, s+\bar{B}+\bar{A}]$. In addition, we have $\bar{W} \leq \bar{A}$, which implies $b \leq \bar{A}$. Since $(s+b, s+b+\bar{B}] \subset[s, s+\bar{B}+\bar{A}]$, $H_{0}$ is able to receive $H_{1}$ 's beacon.
Now we show that $H_{1}$ is able to receive $H_{0}$ 's beacon. Let $\epsilon^{\prime}=$ $\bar{B}-\epsilon$. Since $0 \leq \epsilon \leq \bar{B}$, we have $0 \leq \epsilon^{\prime} \leq \bar{B}$. Suppose $\epsilon^{\prime}=0$, again, from definition of the quorum schemes we know there must exist a Qa-BI, named $q$, on $H_{1}$ that intersects with some Qa-BI on $H_{0}$ [Fig. 11 (2)]. Following the previous discussion with $\epsilon$ substituted by $\epsilon^{\prime}$, we obtain the proof.

\section{REFERENCES}

[1] "The Network Simulator (ns-2)," [Online]. Available: http://www.isi. edu/nsnam $/ \mathrm{ns}$

[2] M. Anand, E. Nightingale, and J. Flinn, "Self-tuning wireless network power management," Wireless Netw., vol. 11, no. 4, pp. 451-469, 2005.

[3] P. Bernstein, V. Hadzilacos, and N. Goodman, Concurrency Control and Recovery in Database Systems. Reading, MA, USA: AddisonWesley, 1987.

[4] G. Bianchi, "Performance analysis of the IEEE 802.11 distributed coordination function," IEEE J. Sel. Areas Commun., vol. 18, no. 3, pp. 535-547, Mar. 2000.

[5] C. Chao, J. Sheu, and I. Chou, "An adaptive quorum-based energy conserving protocol for IEEE 802.11 ad hoc networks," IEEE Trans. Mobile Comput., vol. 5, no. 5, pp. 560-570, May 2006.

[6] B. Choi and X. Shen, "Adaptive asynchronous sleep scheduling protocols for delay tolerant networks," IEEE Trans. Mobile Comput., vol. 10, no. 9, pp. 1283-1296, Sep. 2011.

[7] Z. Chou, "Optimal adaptive power management protocols for asynchronous wireless ad hoc networks," in Proc. IEEE WCNC, 2007, pp. $61-65$.

[8] Y. Chow and H. Teicher, Probability Theory: Independence, Interchangeability, Martingales. New York, NY, USA: Springer-Verlag, 2003.

[9] D. Collins, Carrier Grade Voice Over IP. New York, NY, USA: McGraw-Hill, 2003.

[10] I. L. S. Committee, "Wireless LAN medium access control and physical layer specifications," 1999.

[11] L. Feeney and M. Nilsson, "Investigating the energy consumption of a wireless network interface in an ad hoc networking environment," in Proc. IEEE INFOCOM, 2001, pp. 1548-1557.

[12] J. Jiang, Y. Tseng, C. Hsu, and T. Lai, "Quorum-based asynchronous power-saving protocols for IEEE 802.11 ad hoc networks," Mobile Netw. Appl., vol. 10, no. 1-2, pp. 169-181, 2005.

[13] E. Jung and N. Vaidya, "An energy efficient MAC protocol for wireless LANs," in Proc. IEEE INFOCOM, 2002, pp. 1756-1764.

[14] R. Krashinsky and H. Balakrishnan, "Minimizing energy for wireless Web access with bounded slowdown," Wireless Netw., vol. 11, no. 1-2, pp. $135-148,2005$.

[15] R. Kravets and P. Krishnan, "Application-driven power management for mobile communication," Wireless Netw., vol. 6, no. 4, pp. 263-277, 2000.

[16] D. Logothetis, V. Mainkar, and K. Trivedi, "Transient analysis of nonMarkovian queues via Markov regenerative processes," in Probability and Statistics: AJ Medhi Festschrift. New Delhi, India: New Age Int., 1996.

[17] W. Luk and T. Wong, "Two new quorum based algorithms for distributed mutual exclusion," in Proc. ICDCS, 1997, pp. 100-106.

[18] A. Markopoulou, F. Tobagi, and M. Karam, "Assessing the quality of voice communications over internet backbones," IEEE/ACM Trans. Netw., vol. 11, no. 5, pp. 747-760, Oct. 2003.

[19] K. Medepalli and F. Tobagi, "Towards performance modeling of IEEE 802.11 based wireless networks: A unified framework and its applications," in Proc. IEEE INFOCOM, 2006, pp. 1-12.

[20] A. Papoulis, Probability, Random Variables and Stochastic Processes. New York, NY, USA: McGraw-Hill, 1984.

[21] C. Perkins and E. Royer, "Ad-hoc on-demand distance vector routing," in Proc. IEEE WMCSA, 1999, pp. 90-100.

[22] Y. Tseng, C. Hsu, and T. Hsieh, "Power-saving protocols for IEEE 802.11-based multi-hop ad hoc networks," in Proc. IEEE INFOCOM, 2002, pp. 200-209.

[23] R. Wolff, "Poisson arrivals see time averages," Oper. Res., vol. 30, no 2, pp. 223-231, 1982

[24] W. Ye, J. Heidemann, and D. Estrin, "Medium access control with coordinated adaptive sleeping for wireless sensor networks," IEEE/ACM Trans. Netw., vol. 12, no. 3, pp. 493-506, Jun. 2004. 
[25] R. Zheng, "Design, analysis, and empirical evaluation of power management in multihop wireless networks," Tech. Rep. UIUCDCS-R2003-2381, 2003.

[26] R. Zheng, J. Hou, and L. Sha, "Optimal block design for asynchronous wake-up schedules and its applications in multihop wireless networks," IEEE Trans. Mobile Comput., vol. 5, no. 9, pp. 1228-1241, Sep. 2006.

[27] R. Zheng, J. Hou, and L. Sha, "Performance analysis of power management policies in wireless networks," IEEE Trans. Wireless Commun., vol. 5, no. 6, pp. 1351-1361, Jun. 2006.

[28] R. Zheng and R. Kravets, "On-demand power management for ad hoc networks," in Proc. IEEE INFOCOM, 2003, pp. 481-491.

[29] Z. T. Chou, "A randomized power management protocol with dynamic listen interval for wireless ad hoc networks," in Proc. IEEE VTC-Spring, 2006, pp. 1251-1255.

[30] S. H. Wu, C. M. Chen, and M. S. Chen, "Technical report: Fully adaptive power saving protocols for ad hoc networks using hyper quorum systems," 2007 [Online]. Available: http://telcordia.com/tw/tr/ 1859995682-full.pdf

[31] C. Estan and G. Varghese, "New directions in traffic measurement and accounting: Focusing on the elephants, ignoring the mice," Trans. Comput. Syst., vol. 21, no. 3, pp. 270-313, 2003.

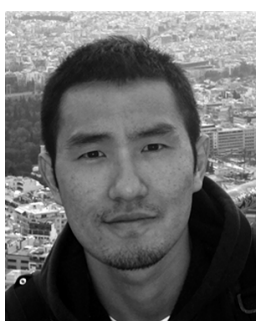

Shan-Hung $\mathrm{Wu}$ received the B.S. degree in information management from National Central University, Jhongli, Taiwan, in 2003, and the M.S. degree in computer science and information and $\mathrm{Ph} . \mathrm{D}$. degree in electrical engineering from National Taiwan University, Taipei, Taiwan, in 2005 and 2009 , respectively.

$\mathrm{He}$ is currently an Assistant Professor with the Department of Computer Science, National Tsing Hua University, Hsinchu, Taiwan. Before joining National Tsing Hua University, he was a Senior Research Scientist with Telcordia Technologies and had led several Telcordia projects including the clustered in-memory database, video surveillance workbench, and the rating engine of the Telcordia ISCP platform. His research interests include wireless networks, database systems, data mining, and mobile data management.

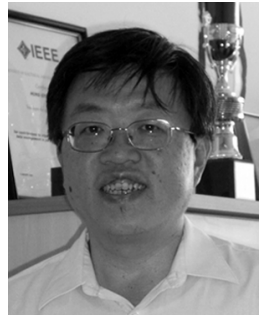

Ming-Syan Chen (M'93-SM'03-F'04) received the B.S. degree in electrical engineering from National Taiwan University, Taipei, Taiwan, and the M.S. and $\mathrm{Ph} . \mathrm{D}$. degrees in computer, information, and control engineering from The University of Michigan, Ann Arbor, MI, USA, in 1985 and 1988, respectively.

$\mathrm{He}$ is now a Distinguished Professor with the Electrical Engineering Department, National Taiwan University. He was a research staff member with the IBM Thomas J. Watson Research Center, Yorktown Heights, NY, USA, from 1988 to 1996; the Director of the Graduate Institute of Communication Engineering, National Taiwan University, from 2003 to 2006; and also the President/CEO of the Institute for Information Industry (III) in Taiwan from 2007 to 2008. He holds, or has applied for, 18 US patents and seven Chinese patents in the areas of data mining, Web applications, interactive video playout, video server design, and concurrency and coherency control protocols. His research interests include database systems, data mining, mobile computing systems, and multimedia networking, and he has published more than 260 papers in his research areas.

Dr. Chen is a Fellow of the Association for Computing Machinery (ACM). In addition to serving as a program committee member in many conferences, he served as an Associate Editor of the IEEE TRANSACTIONS ON KNOWLEDGE AND DATA ENGINEERING from 1997 to 2001, is currently on the Editorial Board of The VLDB Journal, Knowledge and Information Systems, and the International Journal of Electrical Engineering, and is a Distinguished Visitor of the IEEE Computer Society for Asia-Pacific from 1998 to 2000 and also from 2005 to 2007 (invited twice). He served as the International Vice Chair for IEEE INFOCOM 2005, Program Chair of PAKDD 2002, Program Co-Chair of MDM 2003, Program Vice-Chair of IEEE ICDE 2008, CEC/EEE 2006, ICDE 2006 , ICDCS 2005, ICPP 2003, and VLDB 2002, and many other program chairs and co-chairs. He was a keynote speaker on Web data mining in the International Computer Congress in 1999 and IEEE ISM 2007, a tutorial speaker on Web data mining in DASFAA 1999 and on parallel databases in the 11th IEEE ICDE in 1995, and also a Guest Co-Editor for the IEEE TRANSACTIONS ON KNOWLEDGE AND DATA ENGINEERING on a special issue for data mining in December 1996. $\mathrm{He}$ is a recipient of the National Science Council (NSC) Distinguished Research Award, Pan Wen Yuan Distinguished Research Award, Teco Award, Honorary Medal of Information, and K.-T. Li Research Penetration Award for his research work, and also the Outstanding Innovation Award from IBM Corporate for his contribution to a major database product. He also received numerous awards for his research, teaching, inventions, and patent applications.

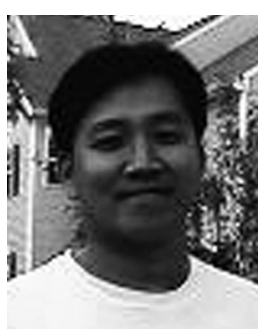

Chung-Min Chen received the B.S. degree from the National Taiwan University, Taipei, Taiwan, in 1985, and the Ph.D. degree from the University of Maryland, College Park, MD, USA, in 1994, both in computer science.

He is currently a Director and Senior Scientist with Telcordia Technologies, Piscataway, NJ, USA. His research interests include data management, network management, and their applications. 